hep-th/0404151

IPM/P-2004/017

\title{
Braneworld inflation
}

\author{
Alex Buchel ${ }^{a, b}$ and Ahmad Ghodsi ${ }^{c}$ \\ ${ }^{a}$ Department of Applied Mathematics \\ University of Western Ontario \\ London, Ontario N6A 5B7, Canada \\ ${ }^{b}$ Perimeter Institute for Theoretical Physics \\ Waterloo, Ontario N2J 2W9, Canada \\ ${ }^{c}$ Institute for Studies in Theoretical Physics and Mathematics (IPM) \\ P.O. Box 19395-5531, Tehran, Iran
}

\begin{abstract}
We discuss various realizations of the four dimensional braneworld inflation in warped geometries of string theory. In all models the inflaton field is represented by a $D p$ probe brane scalar specifying its position in the warped throat of the compactification manifold. We study existing inflationary throat local geometries, and construct a new example. The inflationary brane is either a D3- or a D5-brane of type IIB string theory. In the latter case the inflationary brane is wrapping a two-cycle of the compactification manifold. We discuss some phenomenological aspects of the model where slow-roll conditions are under computational control.
\end{abstract}

April 2004 


\section{Introduction}

Inflation [1-3] is an attractive scenario which solves many important problems in cosmology. The basic idea of its simplest realization is that our Universe went through the stage of the accelerated expansion driven by the potential energy of the slowly rolling inflaton field. In agreement with current observational data such a model naturally predicts a flat Universe and a scale invariant spectrum of density perturbations, provided the inflaton potential is sufficiently flat. It is thus important to find an embedding of inflation in the fundamental theory of quantum gravity, such as a string theory.

Recently there has been considerable progress in implementing this program. Based of the developments of the moduli stabilization problem in string compactifications $[4,5]$, a framework of constructing de-Sitter vacua in string theory (with all moduli stabilized) was proposed in [6] (KKLT). It was further pointed out in [7] $\left(\mathrm{K}^{2} \mathrm{LM}^{2} \mathrm{~T}\right)$ that warped de-Sitter vacua of KKLT is a natural set-up to embed $D 3 \overline{D 3}$ inflation [8-11] into string theory. In the original brane-world model scenarios [8-11] the inflaton field is identified with the separation between four-dimensional domain walls (3-branes) moving in a flat transverse six-dimensional space. The main result of [5] is that in realistic string theory compactifications with stabilized moduli, the six-dimensional compactification manifold is not flat - rather, it must contains one (or more) 'throat' regions with large warp-factors. These warped throat geometries provide string theory realization of the Randall-Sundrum 'compactification' scenario [12]. The authors of [7] studied brane-anti-brane inflation in warped throat geometries. As the $\overline{D 3}$ brane is stabilized at the end of the throat [7], the four-dimensional inflaton field (the $D 3-\overline{D 3}$ brane separation in [8-11] ) can be identified with the position of the $D 3$ in the throat geometry. Unfortunately, the slow roll parameter associated with the $\phi$-field inflation is too large for this model to be realistic

$$
\eta \equiv \frac{1}{3} \frac{V_{\text {inf }}(\phi)^{\prime \prime}}{H^{2}}=\frac{m_{\phi}^{2}}{3 H^{2}}=\frac{2}{3},
$$

where $V_{\text {inf }}(\phi)$ is the inflaton potential, $m_{\phi}^{2}$ is an inflaton mass, and $H$ is the Hubble

scale of the de-Sitter vacua. Above conclusion can be best understood by noting that the inflaton of [7] has an effective four dimensional description in terms of a conformally coupled scalar in the de-Sitter background with a Hubble scale $H$. It was suggested [7] that the $\eta$-problem might be alleviated once the $\phi$-dependence of the overall Kähler modulus of the compactification manifold in the superpotential is taken into account, or if a Kähler stabilization mechanism (as opposite to the superpotential 
stabilization) is used to fix the size of the compactification manifold. Each of these proposed mechanisms is fairly difficult to implement/verify in the context of the lowenergy effective description used to construct de-Sitter vacua of [6].

A complementary approach for analyzing inflation in warped de-Sitter string theory geometries which, in particular, bypasses the difficulties of computing corrections to $\eta$ from the effective four dimensional perspective mentioned above was proposed in [13]. It was pointed out that the brane inflation in the scenario of [7] occurs deep inside the warped throat geometries, where the details of the compactification manifold are not important. All that matters from the compactification manifold is that it, providing a UV completion of the otherwise infinite throat, supplies a four dimensional Hubble parameter $H$. Also, in this setup it is assumed that all moduli of the compactification manifold are fixed, and the scale of moduli stabilization $E_{s}$ is much higher than the relevant scales of inflation $E_{s} \gg H, E_{s} \gg|\phi|$. It is clear that $D 3$ brane inflation in this class of models is equivalent to the probe brane dynamics in the local geometry where the throat, rather then terminating on some complicated (compact) CalabiYau manifold, extends to infinity. The advantage of this viewpoint is that, unlike compact KKLT backgrounds, the corresponding local models can be rather easily and explicitly constructed. For example, much like KS model [14] is a local description of the throat geometry of the GKP compactification [5], the de-Sitter deformed KT model $[15]$ described in $[16,17]$ is a local realization of the throat geometry of the KKLT model $^{1}$. The inflation, or equivalently the brane probe dynamics, can now be studied very explicitly and analytically. Thus, studying inflation as a probe dynamics in deSitter deformed KT backgrounds $[16,17]$ it was shown that the $\eta$-problem persists ${ }^{2}$. It was further shown in [18] that (1.1) is a direct consequence of imaginary-self-dual (ISD) condition ${ }^{3}$ on the 3 -form fluxes, used in $[5,6]$ to stabilize the complex structure moduli of the compactification manifold.

As emphasized in [13], using a probe brane dynamics as a tool for a quantitative

\footnotetext{
${ }^{1}$ Strictly speaking, the correct local model would be de-Sitter deformation of the Klebanov-Strassler solution [14]. For the inflation occurring far from the end of the KS throat the difference between $\mathrm{KT}$ and KS models is subdominant, as it will be for their corresponding de-Sitter deformations. KS de-Sitter deformation as proposed in [17] can be explicitly constructed.

${ }^{2}$ The computations of [7] leading to (1.1) where done in approximation where the 3 -form fluxes of the background geometry are neglected.

${ }^{3}$ The ISD condition is modified in the presence of the supersymmetry breaking effects [19].
} 
analysis of the braneworld inflation ${ }^{4}$ in the warped de-Sitter geometries is quite general, and can be applied outside the inflationary scenario of [7]. Specifically, the warped throat geometry of the inflationary scenario of [7] is locally $A d S_{5}$. The latter is just a reflection of a particular set of fluxes that are turned on. Turning on more generic fluxes would lead to the deformation of the inflationary throat geometry away from being locally $A d S_{5}$. One can imagine that the $\eta$-problem (1.1) in $\mathrm{K}^{2} \mathrm{LM}^{2} \mathrm{~T}$ inflation is a consequence of a quite restrictive set of fluxes used there, and can be alleviated for a judicious choice of fluxes. In fact, it was argued in [18] that a D3 brane inflation in appropriately deformed $A d S_{5}$ local throat geometries can lead to a slow roll inflation. In this paper we confirm that expectation. Additionally, we study 'wrapped-brane' inflationary models. Thus, in section 3 we discuss inflation modeled by a $D 5$ brane wrapped on a two cycle of the de-Sitter deformed Maldacena-Nunez (MN) geometry [21]. The supersymmetric background geometry of MN realizes the backreaction of a large number of $D 5$-branes wrapping a two-cycle of the resolved conifold, with a "twist" preserving four supercharges. The corresponding de-Sitter deformed geometry was explained in details in [23]. Unfortunately we find that from the phenomenological perspective this inflationary model is not viable, as it leads to the slow roll parameter $\eta=\frac{3}{2}$. Next, we study $D 5$ brane inflation in a closely related model, i.e., de-Sitter deformed background of [22] (GKMW). Supersymmetric GKMW solution represents a supergravity description of $D 5$ branes wrapping an $S^{2}$, with the twist preserving eight supercharges. In section 4 we first construct de-Sitter deformation of the GKMW background, and then proceed to the probe brane analysis. As in the case of inflation in the de-Sitter deformed MN throat we find that slow roll inflation in not possible: $\eta \geq 1$. Some phenomenological constraints for the inflationary models are discussed in section 5. The common feature of all discussed local de-Sitter deformed geometries is the presence of an energy scale $\mu$ that breaks conformal invariance characteristic to $A d S_{5}$ throat geometries. Interestingly, depending on the ratio $\mu / H$ certain local geometries undergo 'cosmological phase transitions'. For a local model both $\mu$ and $H$ are nondynamical (parameters). This is not so once local throat geometries are embedded into a global geometry (a compactification). It is possible that these phase transitions might have observable effect on the realistic four dimensional inflation.

Before we move to a somewhat technical discussion of brane probes in de-Sitter deformed local geometries, we would like to mention a phenomenological motivation un-

\footnotetext{
${ }^{4}$ Related ideas were discussed previously in [20].
} 
derling this study. Consider a string theory compactified on a smooth six-dimensional manifold. The presence of $D$-branes will deform a locally flat geometry of a compactification manifold to a warped throat geometry [24,25]. Generically, we expect multiple throats produced from multiple stacks of branes on a compactification manifold. We can imagine a scenario, where one of the throats is of the KKLT type, with a $\overline{D 3}$ brane at the bottom, generating the four dimensional Hubble constant $H$. Though slow roll inflation in that throat is not possible, it might still be realized by a mobile brane in a different throat, which local geometry permits sufficiently flat probe brane potentials ${ }^{5}$. Finally, our proposal is just one way to alleviate the $\eta$-problem. Interesting alternative ideas for overcoming the difficulties described in [7] for string theory inflationary models were presented in [26-34].

\section{Inflation in de-Sitter deformed $\mathcal{N}=2^{*}$ throats}

In [18] it was argued that brane inflation in de-Sitter deformed $\mathcal{N}=2^{*}$ throats might lead to slow roll inflation with arbitrarily small $\eta$ parameter. In this section we provide numerical analysis supporting that claim. The relevant throat geometry is that of the supergravity dual to $\mathcal{N}=2^{*}$ supersymmetric gauge theory constructed in [36] (PW). The probe dynamics in PW background was discussed in details in $[37,38]$. The de-Sitter deformation of the PW geometry was constructed in [39], and the D3 brane probe dynamics was analyzed in [18]. We first review the necessary data for the background geometry and the $D 3$ probe brane effective action. Then we identify singularity-free de-Sitter deformed flows in which the $D 3$ braneworld inflation is slow roll. Phenomenological aspects of the inflation in $\mathcal{N}=2^{*}$ throats are further discussed in section 5.2.

\subsection{The background and the probe brane dynamics}

It is convenient to construct first the background geometry in five-dimensional gauged supergravity, and then further uplift the solution to ten dimensions [39]. The effective five-dimensional action is

$$
S=\int d \xi^{5} \sqrt{-g}\left(\frac{1}{4} R-3(\partial \alpha)^{2}-(\partial \chi)^{2}-\mathcal{P}\right),
$$

\footnotetext{
${ }^{5}$ We will discuss this in some details in the phenomenology section.
} 
where the potential $\mathcal{P}$ is ${ }^{6}$

$$
\mathcal{P}=\frac{1}{48}\left(\frac{\partial W}{\partial \alpha}\right)^{2}+\frac{1}{16}\left(\frac{\partial W}{\partial \chi}\right)^{2}-\frac{1}{3} W^{2},
$$

with the superpotential

$$
W=-e^{-2 \alpha}-\frac{1}{2} e^{4 \alpha} \cosh (2 \chi) .
$$

The supergravity scalars $\alpha$ and $\chi$ encode the renormalization group flow of the $\mathcal{N}=4$ Yang-Mills deformation induced by generically different masses to the bosonic and fermionic components of the $\mathcal{N}=2$ hypermultiplet. To be more specific, we choose the $5 \mathrm{~d}$ RG flow metric as

$$
d s_{5}^{2}=e^{2 A}\left(d S_{4}\right)^{2}+d \rho^{2},
$$

where $\left(d S_{4}\right)^{2}$ is a metric of the four-dimensional de-Sitter space-time with Hubble scale $H=1$. Assuming $A \equiv A(\rho)$ and $\alpha \equiv \alpha(\rho), \chi \equiv \chi(\rho)$, equations of motion (derived from (2.1)) become

$$
\begin{aligned}
& 0=\alpha^{\prime \prime}+4 A^{\prime} \alpha^{\prime}-\frac{1}{6} \frac{\partial \mathcal{P}}{\partial \alpha}, \\
& 0=\chi^{\prime \prime}+4 A^{\prime} \chi^{\prime}-\frac{1}{2} \frac{\partial \mathcal{P}}{\partial \chi}, \\
& \frac{1}{4} A^{\prime \prime}+\left(A^{\prime}\right)^{2}-\frac{3}{4} e^{-2 A}=-\frac{1}{3} \mathcal{P}, \\
& -A^{\prime \prime}-\left(A^{\prime}\right)^{2}=3\left(\alpha^{\prime}\right)^{2}+\left(\chi^{\prime}\right)^{2}+\frac{1}{3} \mathcal{P} .
\end{aligned}
$$

Lacking exact analytical solution of (2.5), in the next section we turn to its numerical analysis. The most general singularity-free solution in the IR $(\rho \rightarrow 0)$ is specified by two parameters $\rho_{0}, \chi_{0}$

$$
\begin{aligned}
e^{A} & =\rho\left(1+\rho^{2}\left(\frac{1}{72} \rho_{0}^{-4}+\frac{1}{36} \rho_{0}^{2} \cosh \left(2 \chi_{0}\right)-\frac{1}{288} \rho_{0}^{8} \sinh ^{2}\left(2 \chi_{0}\right)\right)+\mathcal{O}\left(\rho^{4}\right)\right), \\
e^{\alpha} & =\rho_{0}+\rho^{2}\left(\frac{1}{60} \rho_{0}^{-3}-\frac{1}{60} \rho_{0}^{3} \cosh \left(2 \chi_{0}\right)+\frac{1}{120} \rho_{0}^{9} \sinh ^{2}\left(2 \chi_{0}\right)\right)+\mathcal{O}\left(\rho^{4}\right), \\
\chi & =\chi_{0}+\rho^{2}\left(-\frac{1}{20} \rho_{0}^{2} \sinh \left(2 \chi_{0}\right)+\frac{1}{160} \rho_{0}^{8} \sinh \left(4 \chi_{0}\right)\right)+\mathcal{O}\left(\rho^{4}\right) .
\end{aligned}
$$

The ultraviolet $(\rho \rightarrow \infty)$ asymptotics are conveniently written in terms of a new radial coordinate

$$
x \equiv e^{-\rho / 2} .
$$

\footnotetext{
${ }^{6}$ We set the 5d gauged SUGRA coupling to one. This corresponds to setting $S^{5}$ radius $L=2$.
} 
We find

$$
\begin{aligned}
& A=-\ln x-x^{2}\left(e^{-2 \xi}+\frac{1}{3} \chi_{00}^{2}\right)+x^{4}\left(\frac{1}{9} \chi_{00}^{4}-\frac{1}{2} e^{-4 \xi}+\frac{1}{6} \chi_{00}^{2} e^{-2 \xi}-\frac{1}{2} \chi_{00}^{2} \chi_{10}-\rho_{10}^{2}-\frac{1}{8} \rho_{11}^{2}\right. \\
&-\left.\left(2 \chi_{00}^{2} e^{-2 \xi}+\frac{2}{3} \chi_{00}^{4}+2 \rho_{10} \rho_{11}\right) \ln x-\rho_{11} \ln ^{2} x\right)+\mathcal{O}\left(x^{6} \ln ^{3} x\right) \\
& e^{\alpha}=+x^{2}\left(\rho_{10}+\rho_{11} \ln x\right)+x^{4}\left(\frac{1}{3} \chi_{00}^{4}+\frac{3}{2} \rho_{10}^{2}-2 \rho_{10} \rho_{11}+\frac{3}{2} \rho_{11}^{2}+\frac{2}{3} \chi_{00}^{2}\left(5 \rho_{10}-4 \rho_{11}\right)\right. \\
&+2 e^{-2 \xi}\left(2 \rho_{10}-\rho_{11}\right)+\left(\frac{10}{3} \chi_{00}^{2} \rho_{11}+3 \rho_{10} \rho_{11}-2 \rho_{11}^{2}+4 \rho_{11} e^{-2 \xi}\right) \ln x \\
&\left.+\frac{3}{2} \rho_{11}^{2} \ln ^{2} x\right)+\mathcal{O}\left(x^{6} \ln ^{3} x\right) \\
& \\
& \quad \chi=\chi_{00} x\left(1+x^{2}\left(\chi_{10}+\left(\frac{4}{3} \chi_{00}^{2}+4 e^{-2 \xi}\right) \ln x\right)\right)+\mathcal{O}\left(x^{5} \ln ^{2} x\right)
\end{aligned}
$$

where $\left\{\xi, \chi_{00}, \chi_{10}, \rho_{10}, \rho_{11}\right\}$ are parameters characterizing the ultraviolet asymptotics, and are functions of the infrared data $\left\{\rho_{0}, \chi_{0}\right\}$. As explained in [40], $\rho_{11}\left(\chi_{00}\right)$ should be identified with the mass $m_{b}^{2}\left(m_{f}\right)$ of the bosonic (fermionic) components of the $\mathcal{N}=2$ hypermultiplet. Two more parameters $\rho_{10}, \chi_{10}$ are related to the bosonic and fermionic bilinear condensates correspondingly. Finally, $\xi$ is a residual integration constant associated with fixing the radial coordinate - it can be removed at the expense of shifting the origin of the radial coordinate $\rho$, or rescaling $x$. As the origin of the radial coordinate is 'fixed' in specifying the infrared boundary conditions (2.6), $\xi \equiv \xi\left(\rho_{0}, \chi_{0}\right)$.

The complete ten-dimensional lift of the RG flow (2.5) was presented in [39], and the $D 3$ brane probe dynamics in the resulting 10d geometry was studied in [18]. Identifying the inflaton with the radial motion of the probe brane in this background geometry, the inflaton mass $m_{\phi}^{2}$ was found to be

$$
m_{\phi}^{2}=2+\left[\frac{2}{3} e^{2 \xi} \chi_{00}^{2}\right]+\left[e^{2 \xi} \rho_{11}\left(\frac{3}{2} \cos ^{2} \theta-1\right)\right],
$$

thus leading to inflationary slow roll parameter

$$
\eta=\frac{2}{3}+\left[\frac{2}{9} e^{2 \xi} \chi_{00}^{2}\right]+\left[\frac{1}{3} e^{2 \xi} \rho_{11}\left(\frac{3}{2} \cos ^{2} \theta-1\right)\right] .
$$


Few comments about (2.11), (2.12) are in order.

- Inflaton mass depends on one of the angles $(\theta)$ of the squashed $S^{5}$ in the tendimensional background [18];

- Turning off the mass deformation (setting $\rho_{0}=\chi_{0}=0$ ) gives rise to $m_{\phi}^{2}=2$, which is the effective mass of the conformally coupled scalar of the $\mathcal{N}=4$ vector multiplet;

- Turning on mass to the fermionic components of the $\mathcal{N}=2$ hypermultiplet always raises the inflaton mass. Thus the slow-roll inflation is most effectively generated with $\chi_{00}=0$. Actually, $\chi(\rho) \equiv 0$ is an exact solution of $(2.5)$, which we restrict to from now on.

- Recall that bosonic mass deformation parameter $\rho_{11} \propto m_{b}^{2}$. In principle, in the supergravity solution $\rho_{11}$ can be either positive or negative. However, without a stabilizing effect of the gauge theory background curvature (setting $H \rightarrow 0$ or removing '2' in (2.11)), $\rho_{11}<0$ would lead to the supergravity background instabilities associated with unboundedness of the probe brane potential close to the boundary. This is a reflection of the dual gauge theory instabilities corresponding to $m_{b}^{2}<0$. Once $H \neq 0$, sufficiently small negative $m_{b}^{2}$ will not destabilize the background:

$$
0 \geq e^{2 \xi} \rho_{11} \geq-4
$$

In the regime (2.13), a $D 3$ probe would tend to move in the $\cos ^{2} \theta=1$ 'valley', where its potential energy is locally minimized, leading to a slow-roll parameter $\eta \equiv \eta_{-}(2.12)$

$$
\eta_{-}=\frac{2}{3}+\frac{1}{6} e^{2 \xi} \rho_{11}, \quad \eta_{-} \in\left[0, \frac{2}{3}\right]
$$

In the case of $\rho_{11}>0$ (a positive $m_{b}^{2}$ ), the $D 3$ probe brane potential energy is minimum in the $\cos \theta=0$ valley $^{7}$. Here background stability against spontaneous $D 3 \bar{D} 3$-pair production constrains

$$
2 \geq e^{2 \xi} \rho_{11} \geq 0
$$

leading to a slow-roll parameter $\eta \equiv \eta_{+}$

$$
\eta_{+}=\frac{2}{3}-\frac{1}{3} e^{2 \xi} \rho_{11}, \quad \eta_{+} \in\left[0, \frac{2}{3}\right]
$$

\subsection{Slow roll inflation}

We now map numerically the phase space of the $D 3$ inflation in de-Sitter deformed $\mathcal{N}=2^{*}$ throat reviewed in the previous section. The procedure is to numerically

\footnotetext{
${ }^{7}$ For $H=0$ this submanifold is a moduli space of a $D 3$ probe in the PW background $[37,38]$.
} 
integrate (2.5) from the infrared $(\rho=0)(2.6)$ to the ultraviolet $(2.8),(2.9)(\rho \rightarrow \infty)$, and, given ${ }^{8}\left\{\rho_{0}\right\}$ in the IR extract $\left\{\xi, \rho_{11}\right\}$ in the UV. Depending on the sign of $\rho_{11}$, we can use $(2.14),(2.16)$ to determine $\eta_{ \pm}$. Though simple to state, the problem is rather challenging to implement. The reason is the huge exponential asymptotic suppression of the coefficient $\rho_{11},(2.9)$. Ultimately, we resolved this technical difficulty by reparameterizing $\alpha(\rho)$ as follows

$$
e^{\alpha} \equiv 1+\frac{B(\rho)}{1+e^{2 A(\rho)}},
$$

and rewriting (2.5) in terms of $A(\rho)$ and $B(\rho)$ with $\chi(\rho) \equiv 0$. Notice that such a redefinition implies identification

$$
\begin{aligned}
\rho_{0} & \equiv \lim _{\rho \rightarrow 0}(1+B(\rho)), \\
e^{2 \xi} \rho_{11} & \equiv-2 \lim _{\rho \rightarrow \infty} \frac{d B(\rho)}{d \rho} .
\end{aligned}
$$

Obviously, since $e^{\alpha} \geq 0, \rho_{0} \geq 0$, and thus $B(\rho=0) \geq-1$.

Results of numerical integration are presented in Fig. 1-Fig. 3. For $\rho_{0}=1$ we have undeformed $A d S_{5}$ throat, leading to a familiar result for the slow roll parameter $\eta=\frac{2}{3}$. We find that for $0 \leq \rho_{0} \leq 1, \rho_{11}\left(\rho_{0}\right) \geq 0$, thus the corresponding slow roll parameter is $\eta \equiv \eta_{+}$, defined by (2.16), Fig. 2. For $1<\rho_{0} \leq \rho_{\text {critical }} \approx 1.2$, we find $\rho_{11}\left(\rho_{0}\right) \leq 0$, thus the corresponding slow roll parameter is $\eta \equiv \eta_{-}$, defined by (2.14), Fig. 1. As $\rho_{0}>\rho_{\text {critical }}$, the inflaton mass $m_{\phi}^{2}<0$. In this case rather that moving inside the warped throat (toward the infrared end), the probe brane will move to the boundary, with its potential energy being unbounded from below. If the spatial directions of the probe brane are compactified, then the background will have non-perturbative instability with respect to the spontaneous brane-anti-brane creation [41].

\section{Inflation in de-Sitter deformed MN background}

Typically an inflaton of a brane inflationary scenario in string theory is identified with a scalar coordinate of a 3-brane. This is the case, in particular, for the inflationary model of [7], and the model discussed in the previous section. Since string theory compactification manifold might contain topologically non-trivial cycles, one might wonder whether a more exotic inflationary scenario might be slow roll. Specifically [13],

\footnotetext{
${ }^{8}$ We explained in the previous section that the most efficient inflation occurs for $\chi_{0}=0$.
} 
one can imagine inflation realized by a probe $D p$-brane, for $p>3$, wrapping a $(p-3)$ cycle of the compactification manifold. In the following two sections we study inflation from D5-branes wrapping a two-cycle of a local de-Sitter deformed geometry. We begin with inflation modeled by a $D 5$ brane wrapped on a two cycle of de-Sitter deformed MN geometry [21].

After reviewing the construction of the background [23], we study D5-probe dynamics. Unfortunately, the slow-roll inflation is not possible in this model. For a canonically normalized inflaton we find

$$
\eta_{M N}=\frac{3}{2}
$$

\subsection{The background}

The de-Sitter deformation of the MN supergravity background was constructed and studied in details in [23]. Here, the string frame metric is

$$
d s_{s t}^{2}=F^{2}\left(d s_{\mathcal{M}_{4}}\right)^{2}+n\left(d \rho^{2}+G^{2} d \Omega_{2}^{2}+\frac{1}{4} \sum_{a}\left(\omega_{a}-A_{a}\right)^{2}\right)
$$

where $\Omega_{2}^{2}$ is a round $S^{2}$ (parameterized by $\left.(\tilde{\theta}, \tilde{\phi})\right)$ which the branes wrap,

$$
\left(d s_{\mathcal{M}_{4}}\right)^{2}(x) \equiv-d t^{2}+\frac{1}{H^{2}} \cosh ^{2} H t d \Omega_{3}^{2} .
$$

and $\omega_{a}$ are the $S U(2)$ left-invariant one forms on the $S^{3}$ (parametrized by $(\theta, \phi, \psi)$ ) transverse to the NS5-branes,

$$
\begin{aligned}
& \omega_{1}=\cos \phi d \theta+\sin \phi \sin \theta d \psi, \\
& \omega_{2}=-\sin \phi d \theta+\cos \phi \sin \theta d \psi, \\
& \omega_{3}=d \phi+\cos \theta d \psi .
\end{aligned}
$$

Also in (3.2), $A_{a}$ are the $S U(2)_{R}$ gauge fields on the $S^{2}$ realizing the twist,

$$
\begin{aligned}
& A_{1}=a d \tilde{\theta} \\
& A_{2}=a \sin \tilde{\theta} d \tilde{\phi} \\
& A_{3}=\cos \tilde{\theta} d \tilde{\phi}
\end{aligned}
$$

Finally, there is a dilaton $\Phi=\ln g_{s}$, and an NS-NS 3-form flux

$$
H_{3}=n\left[-\frac{1}{4}\left(\omega_{1}-A_{1}\right) \wedge\left(\omega_{2}-A_{2}\right) \wedge\left(\omega_{3}-A_{3}\right)+\frac{1}{4} \sum_{a} F_{a} \wedge\left(\omega_{a}-A_{a}\right)\right]
$$


where $F_{a}=d A_{a}+\frac{1}{2} \epsilon_{a b c} A_{b} \wedge A_{c}$. Altogether, the background is parameterized by four functions $F, G, a, g_{s}$ of the radial coordinate $\rho \in[0,+\infty)$.

With this ansatz, the type IIB supergravity equations of motion for the deformed MN model are reduced to ${ }^{9}$

$$
\begin{aligned}
& 0=\left[\frac{a^{\prime} F^{4}}{g_{s}^{2}}\right]^{\prime}-\frac{a F^{4}\left(a^{2}-1\right)}{g_{s}^{2} G^{2}}, \\
& 0=\left[\frac{\left(G^{2}\right)^{\prime} F^{4}}{g_{s}^{2}}\right]^{\prime}+\frac{F^{4}}{2 g_{s}^{2} G^{2}}\left\{\left(a^{2}-1\right)^{2}+G^{2}\left[\left(a^{\prime}\right)^{2}-4\right]\right\}, \\
& 0=\left[G^{2} F^{4}\left(\frac{1}{g_{s}^{2}}\right)^{\prime}\right]^{\prime}-\frac{F^{4}}{4 g_{s}^{2} G^{2}}\left\{\left(a^{2}-1\right)^{2}+2 G^{2}\left[8 G^{2}+\left(a^{\prime}\right)^{2}\right]\right\}, \\
& 0=\left[\frac{\left(F^{4}\right)^{\prime} G^{2}}{g_{s}^{2}}\right]^{\prime}-\frac{12 n H^{2} F^{2} G^{2}}{g_{s}^{2}} .
\end{aligned}
$$

There is also a first order constraint coming from fixing the re-parametrization invariance (the choice of $\rho$ ),

$$
\begin{aligned}
0= & F^{2}\left\{2 G^{2}\left[8 G^{2}\left(g_{s}^{\prime}\right)^{2}+4 g_{s}^{2}\left(G^{\prime}\right)^{2}-4\left(G^{2}\right)^{\prime}\left(g_{s}^{2}\right)^{\prime}-g_{s}^{2}\left(a^{\prime}\right)^{2}\right]\right. \\
& \left.+g_{s}^{2}\left[\left(a^{2}-1\right)^{2}-8 G^{2}\left(1+2 G^{2}\right)\right]\right\}+16 g_{s} G^{3}\left\{3 g_{s} G\left(\left(F^{\prime}\right)^{2}-n H^{2}\right)\right. \\
& \left.+2\left(F^{\prime}\right)^{2} g_{s}^{2}\left(\frac{G}{g_{s}}\right)^{\prime}\right\} .
\end{aligned}
$$

\subsection{Probe dynamics}

We will study D5 probe dynamics, where the probe brane extends in four de-Sitter directions, and wraps the $\Omega_{2}$ directions. For this we would need to go to S-dual geometry, and compute the pullback of the $\operatorname{RR~} C_{(6)}$ to the brane worldvolume. Note that, performing S-duality we find

$$
\begin{aligned}
g_{s t}^{D 5} & =\frac{1}{g_{s}} \\
d s_{s t}^{2}(D 5) & =g_{s}^{-1} d s_{s t}^{2}(N S 5), \\
C_{(2)} & =-B, \quad C_{(6)}=-\tilde{B},
\end{aligned}
$$

where $d s_{s t}^{2}(N S 5)$ is the string frame metric (3.2), and

$$
d \tilde{B}=g_{s}^{-2} \star H_{3},
$$

\footnotetext{
${ }^{9}$ The prime denotes derivative with respect to $\rho$.
} 
where the Hodge dual is taken in NS5 metric (3.2). Explicitly, we find

$$
\begin{aligned}
\tilde{B}=\operatorname{vol}_{\mathcal{M}_{4}} \wedge & {\left[\mathcal{V}_{1}(\rho) \sin \tilde{\theta} d(\tilde{\theta}) \wedge d(\tilde{\phi})+\mathcal{V}_{2}(\rho) \sin \theta d(\psi) \wedge d(\theta)\right.} \\
& \left.+\frac{n F^{4} a^{\prime}}{8 g_{s}^{2}} \mathcal{O}_{2}\right]
\end{aligned}
$$

where $\operatorname{vol}_{\mathcal{M}_{4}}$ is the volume form on $\mathcal{M}_{4}(3.3)$, and

$$
\begin{aligned}
\mathcal{V}_{1}^{\prime}= & \frac{n F^{4}\left(a^{4}-a^{2}-16 G^{4}\right)}{8 g_{s}^{2} G^{2}}, \\
\mathcal{V}_{2}^{\prime}= & \frac{n F^{4}\left(1-a^{2}\right)}{8 g_{s}^{2} G^{2}}, \\
\mathcal{O}_{2}= & \sin \tilde{\theta} \sin \theta \sin \phi d(\tilde{\phi}) \wedge d(\psi)+\sin \theta \cos \phi d(\psi) \wedge d(\tilde{\theta}) \\
& +\sin \tilde{\theta} \cos \phi d(\tilde{\phi}) \wedge d(\theta)+\sin \phi d(\tilde{\theta}) \wedge d(\theta),
\end{aligned}
$$

where again, primes denote derivatives with respect to $\rho$. Notice that only the first term in (3.11) survives the pullback to the worldvolume of the $D 5$ probe.

Consider a $D 5$ probe with the worldvolume $\mathcal{M}_{4} \times S^{2}$, where $S^{2}$ is parameterized by $(\tilde{\theta}, \tilde{\phi})$ in $(3.2)$. The probe brane action reads [42]

$$
S_{D 5}=-\mu_{5} \int_{\mathcal{M}_{4} \times S^{2}} d^{6} \xi \frac{1}{g_{s}^{D 5}} \sqrt{-g_{D 5}}+\mu_{5} \int_{\mathcal{M}_{4} \times S^{2}} C_{(6)},
$$

where $g_{D 5}$ is the pullback of the $d s_{s t}(D 5)$ (3.9) to the probe brane worldvolume, and $C_{(6)}$ is given by (3.9)-(3.12). For a slowly moving probe in $\rho \equiv \rho\left(\mathcal{M}_{4}\right)$ direction, localized at a point in $S^{3}$, we find the effective action $S_{\rho}$

$$
\begin{aligned}
S_{\rho}= & \int_{\mathcal{M}_{4}} d^{4} x \sqrt{-g_{\mathcal{M}_{4}}}\left(-\frac{n^{2}\left(4 G^{2}+a^{2}\right) F^{2}}{8 g_{s}^{2}} 4 \pi \mu_{5} E\left(\sqrt{1-\frac{1}{4 G^{2}+a^{2}}}\right) \partial_{\mu} \rho \partial^{\mu} \rho\right. \\
& -\mathcal{V}(\rho))
\end{aligned}
$$

where

$$
\mathcal{V}(\rho)=4 \pi \mu_{5}\left(\frac{n\left(4 G^{2}+a^{2}\right) F^{4}}{4 g_{s}^{2}} E\left(\sqrt{1-\frac{1}{4 G^{2}+a^{2}}}\right)+\mathcal{V}_{1}\right)
$$

and the complete elliptic integral is defined as follows

$$
E(x) \equiv \int_{0}^{1} \sqrt{\frac{1-x^{2} t^{2}}{1-t^{2}}} d t
$$


In what follows we use canonically normalized inflaton $\rho \rightarrow \Phi$

$$
4 \pi \mu_{5} \frac{n^{2}\left(4 G^{2}+a^{2}\right) F^{2}}{4 g_{s}^{2}} E\left(\sqrt{1-\frac{1}{4 G^{2}+a^{2}}}\right) \partial_{\mu} \rho \partial^{\mu} \rho \equiv \partial_{\mu} \Phi \partial^{\mu} \Phi,
$$

leading to

$$
S_{\Phi}=\int_{\mathcal{M}_{4}} d^{4} x \sqrt{-g_{\mathcal{M}_{4}}}\left(-\frac{1}{2} \partial_{\mu} \Phi \partial^{\mu} \Phi-\mathcal{V}(\Phi)\right) .
$$

Asymptotic $\rho \rightarrow \infty$ solution of (3.7) was given in [23]

$$
\begin{aligned}
F & =\left(3 n H^{2} \rho\right)^{1 / 2}+\cdots, \\
G^{2} & =\rho+\cdots, \\
g_{s} & =g_{0}\left(\rho^{3 / 4} e^{-\rho}+\cdots\right), \\
a & =\Upsilon \rho^{-1 / 2}(1+\cdots)+\mathcal{C} \rho^{1 / 2} e^{-2 \rho}(1+\cdots),
\end{aligned}
$$

where $\cdots$ denote corrections which are subdominant as $\rho \rightarrow \infty$. Given (3.19), and the normalization (3.17), we find

$$
\mathcal{V}(\Phi)=\frac{9}{4} H^{2} \Phi^{2}\left(1+\mathcal{O}\left(\ln ^{-1} \Phi\right)\right),
$$

which leads to a slow-roll parameter reported in (3.1). We conclude that the slow roll inflation is not possible in this model.

\section{4 de-Sitter deformed GKMW background}

Our next example of a wrapped braneworld inflationary model is represented by a D5probe brane moving in a de-Sitter deformed local warped throat geometry of GKMW [22]. In the absence of the deformation, $H=0$, GKMW and MN [21] models differ by the supersymmetry preserving twist for a five-brane wrapping a two-cycle of the resolved conifold. Apparently, this difference is not enough to overcome the large- $\eta$ problem. Here we find

$$
\eta_{G K M W} \geq 1
$$

We begin with constructing de-Sitter deformation of the background [22]. We then study the $D 5$ probe brane dynamics, phases of the background geometry, and the slow roll condition. 


\subsection{The background}

The supergravity background corresponding to $N S 5$ branes wrapped on $S^{2}$ with $\mathcal{N}=2$ supersymmetry in four dimensions has been constructed in [22]. In this section we study de-Sitter deformations of this geometry. Following [22] we construct deformed solution in $D=7 S O(4)$ gauged supergravity, and then further uplift it to ten dimensions using [43].

The effective lagrangian of the relevant $D=7$ gauged supergravity reads [22]

$$
\mathcal{L}=\sqrt{-g}\left(R-\frac{5}{16} \partial_{\mu} y \partial^{\mu} y-\partial_{\mu} x \partial^{\mu} x-\frac{1}{4} e^{-2 x-y / 2} F_{\mu \nu}^{(2)} F^{(2) \mu \nu}+4 g^{2} e^{y / 2}\right),
$$

where $x, y$ are scalar fields, and $F_{\mu \nu}^{(2)}$ is a field strength of the $U(1) \subset S O(4)$ gauge fields. For the metric and the gauge field we choose

$$
\begin{aligned}
d s_{7}^{2} & =e^{2 f}\left(F^{2}\left(d s_{\mathcal{M}_{4}}\right)^{2}+d \rho^{2}\right)+a^{2} d \Omega_{2}^{2}, \\
F^{(2)} & =\frac{1}{g} \operatorname{vol}_{\Omega_{2}},
\end{aligned}
$$

where $\left(d s_{\mathcal{M}_{4}}\right)^{2}$ is given by (3.3), and $a, f, F, x, y$ are functions of a radial coordinate $\rho$ only.

We obtain the following equations of motion

$$
\begin{gathered}
x^{\prime \prime}+\left(3 f^{\prime}+2 \frac{a^{\prime}}{a}+4 \frac{F^{\prime}}{F}\right) x^{\prime}=-\frac{1}{2 g^{2} a^{4}} e^{2 f-2 x-y / 2}, \\
y^{\prime \prime}+\left(3 f^{\prime}+2 \frac{a^{\prime}}{a}+4 \frac{F^{\prime}}{F}\right) y^{\prime}=-\frac{1}{5} e^{2 f}\left(\frac{2}{g^{2} a^{4}} e^{-2 x-y / 2}+16 g^{2} e^{y / 2}\right) \\
(f+\ln F)^{\prime \prime}+\left(3 f^{\prime}+4(\ln F)^{\prime}+2 \frac{a^{\prime}}{a}\right)(f+\ln F)^{\prime}-\frac{3 H^{2}}{F^{2}} \\
=\frac{1}{10} e^{2 f}\left(\frac{1}{g^{2} a^{4}} e^{-2 x-y / 2}+8 g^{2} e^{y / 2}\right) \\
4(f+\ln F)^{\prime \prime}+2 \frac{a^{\prime \prime}}{a}-2 \frac{a^{\prime}}{a}(f+\ln F)^{\prime}+2(\ln F)^{\prime}\left(\frac{a^{\prime}}{a}+2(f+\ln F)^{\prime}\right) \\
=\frac{1}{10} e^{2 f}\left(\frac{1}{g^{2} a^{4}} e^{-2 x-y / 2}+8 g^{2} e^{y / 2}\right)-\frac{5}{16}\left(y^{\prime}\right)^{2}-\left(x^{\prime}\right)^{2} \\
\frac{a^{\prime \prime}}{a}+3 \frac{a^{\prime}}{a} f^{\prime}+\frac{\left(a^{\prime}\right)^{2}}{a^{2}}+4 \frac{a^{\prime}}{a}(\ln F)^{\prime} \\
=e^{2 f}\left(\frac{1}{a^{2}}-\frac{2}{5 g^{2} a^{4}} e^{-2 x-y / 2}+\frac{4}{5} g^{2} e^{y / 2}\right)
\end{gathered}
$$




$$
\begin{aligned}
0= & 6 a^{2} f^{\prime}\left(F^{2}\right)^{\prime}+6 a^{2}\left(F^{\prime}\right)^{2}+6 a^{2}\left(f^{\prime}\right)^{2} F^{2}+4\left(a^{2}\right)^{\prime} F^{2} f^{\prime}+2\left(a^{2}\right)^{\prime}\left(F^{2}\right)^{\prime}+\left(a^{\prime}\right)^{2} F^{2}-6 H^{2} a^{2} \\
& -\frac{F^{2}}{32 g^{2} a^{2}}\left(32 a^{2} g^{2} e^{2 f}+5 g^{2} a^{4}\left(y^{\prime}\right)^{2}+16 g^{2} a^{4}\left(x^{\prime}\right)^{2}+64 a^{4} g^{4} e^{2 f+y / 2}-8 e^{2 f-2 x-y / 2}\right) .
\end{aligned}
$$

In (4.4)-(4.9) primes denote derivatives with respect to $\rho$. With $H=0, F \equiv 1$ above equations are the same as in [22]. We explicitly verified that though overdetermined, (4.4)-(4.8) are self-consistent even with $H \neq 0$. As in [22] we can solve for $y$ with

$$
y=-4 f
$$

Consistent Kaluza-Klein reductions on spheres developed in [43] does not rely on supersymmetry. Thus using formula of [43], we can uplift the $D=7$ solution constructed above to a full ten-dimensional solution. We find

$$
\begin{gathered}
d s_{s t}^{2}=F^{2}\left(d s_{\mathcal{M}_{4}}\right)^{2}+d \rho^{2}+a^{2} e^{-2 f} d \Omega_{2}^{2}+\frac{1}{g^{2}} d \theta^{2} \\
+\frac{e^{-x} \cos ^{2} \theta}{g^{2} \Omega}\left(d \phi_{1}+\cos \tilde{\theta} d \tilde{\phi}\right)^{2}+\frac{e^{x} \sin ^{2} \theta}{g^{2} \Omega} d \phi_{2}^{2}, \\
g_{s}^{-2}=e^{5 f} \Omega, \\
H_{3}=\frac{2 \sin \theta \cos \theta}{g^{2} \Omega^{2}}(\sin \theta \cos \theta d x-d \theta) \wedge\left(d \phi_{1}+\cos \tilde{\theta} d \tilde{\phi}\right) \wedge d \phi_{2} \\
+\frac{e^{-x} \sin ^{2} \theta}{g^{2} \Omega} \sin \tilde{\theta} d \tilde{\theta} \wedge d \tilde{\phi} \wedge d \phi_{2},
\end{gathered}
$$

where the metric is given in the string frame, $\left\{\theta, \phi_{1}, \phi_{2}\right\}$ are coordinates parameterizing squashed and twisted $S^{3}$ transverse to wrapped NS5 branes, and

$$
\Omega \equiv e^{x} \cos ^{2} \theta+e^{-x} \sin ^{2} \theta
$$

We explicitly verified that for the background (4.11)-(4.13) ten dimensional type IIB supergravity equations of motion reduce to (4.4)-(4.8). 


\subsection{Probe dynamics}

The computations here parallel those of section 3.2. For the six-form potential Hodge dual to the NSNS 3-form flux (4.13) we find

$$
\begin{aligned}
& \tilde{B}=\operatorname{vol}_{\mathcal{M}_{4} \wedge} {\left[\left(\mathcal{V}_{1}(\rho)+\mathcal{V}_{2}(\rho) \cos ^{2} \theta\right) \sin \tilde{\theta} d(\tilde{\theta}) \wedge d(\tilde{\phi})\right.} \\
&\left.+\mathcal{V}_{3}(\rho) \cos ^{2} \theta \cos \tilde{\theta} d(\rho) \wedge d(\tilde{\phi})+\mathcal{V}_{4}(\rho) \sin ^{2} \theta d(\rho) \wedge d\left(\phi_{1}\right)\right] \\
&=\operatorname{vol}_{\mathcal{M}_{4}} \wedge {\left[\left(\mathcal{V}_{1}(\rho)+\mathcal{V}_{2}(\rho) \cos ^{2} \theta\right) \sin \tilde{\theta} d(\tilde{\theta}) \wedge d(\tilde{\phi})\right.} \\
&\left.+\mathcal{V}_{3}(\rho) \cos ^{2} \theta d(\rho) \wedge\left(\cos \tilde{\theta} d(\tilde{\phi})+d\left(\phi_{1}\right)\right)\right]
\end{aligned}
$$

where $\operatorname{vol}_{\mathcal{M}_{4}}$ is the volume form on $\mathcal{M}_{4}(3.3)$, and

$$
\begin{aligned}
& \mathcal{V}_{1}^{\prime}=-2 a^{2} g F^{4} e^{3 f} \\
& \mathcal{V}_{2}=\frac{F^{4} e^{3 f} a^{2} x^{\prime}}{g}, \\
& \mathcal{V}_{3}=\frac{F^{4} e^{7 f-2 x}}{2 g^{3} a^{2}} \\
& \mathcal{V}_{4}=-\frac{F^{4} e^{7 f-2 x}}{2 g^{3} a^{2}}
\end{aligned}
$$

The second equality in (4.15) is valid up to gauge transformations $\tilde{B} \sim \tilde{B}+d \mathcal{A}$.

As before, we consider a $D 5$ probe brane with the worldvolume $\mathcal{M}_{4} \times S^{2}$. For a slowly moving probe in $\rho \equiv \rho\left(\mathcal{M}_{4}\right)$ direction, localized at a point in $S^{3}$, we find the effective action

$$
S_{\rho}=\mu_{5} \int_{\mathcal{M}_{4} \times S^{2}} d^{4} x \sin \tilde{\theta} d \tilde{\theta} d \tilde{\phi} \sqrt{-g_{\mathcal{M}_{4}}}\left(-\frac{1}{2} \mathcal{K}_{\rho}(\rho) \partial_{\mu} \rho \partial^{\mu} \rho-\mathcal{V}(\rho)\right)
$$

where

$$
\begin{aligned}
& \mathcal{K}_{\rho}=F^{2} a^{2} e^{3 f} \Omega\left(1+\frac{e^{2 f} \cos ^{2} \theta}{g^{2} e^{x} \Omega a^{2} \tan ^{2} \tilde{\theta}}\right)^{1 / 2} \\
& \mathcal{V}=F^{4} a^{2} e^{3 f} \Omega\left(1+\frac{e^{2 f} \cos ^{2} \theta}{g^{2} e^{x} \Omega a^{2} \tan ^{2} \tilde{\theta}}\right)^{1 / 2}+\mathcal{V}_{1}(\rho)+\mathcal{V}_{2}(\rho) \cos ^{2} \theta
\end{aligned}
$$


As a check, we compare with the $\mathcal{N}=2$ supersymmetric flow [22]. Here we have

$$
\begin{aligned}
F & =1, \quad H=0, \\
\frac{d \mathcal{V}_{1}}{d z} & =-2 g^{2} z e^{2 g^{2} z}, \quad \frac{d z}{d \rho}=\frac{1}{g e^{x}}, \\
\mathcal{V}_{2} & =-\frac{z e^{2 g^{2} z}}{2 g^{2}} \frac{d}{d z}\left[e^{-2 x}\right], \\
a^{2} e^{3 f+x} & =z e^{2 g^{2} z}, \quad a^{2} e^{-2 f}=z, \quad e^{-2 x}=1-\frac{1+k e^{-2 g^{2} z}}{2 g^{2} z},
\end{aligned}
$$

leading to a moduli space $\cos \theta=0$ (for all values of the parameter $k$ ), and the kinetic term for $\rho$ on the moduli space

$$
-\frac{1}{2} a^{2} e^{3 f-x} \partial_{\mu} \rho \partial^{\mu} \rho=-\frac{1}{2} g^{2} z e^{2 g^{2} z} \partial_{\mu} z \partial^{\mu} z
$$

in agreement with [22]. Four dimensional effective action from $S_{\rho}(4.17)$ reads

$$
\begin{aligned}
S_{\rho}= & \int_{\mathcal{M}_{4}} d^{4} x \sqrt{-g_{\mathcal{M}_{4}}}\left(-\frac{a^{2} F^{2} e^{3 f} \Omega}{2} 4 \pi \mu_{5} E\left(\sqrt{1-\frac{e^{2 f} \cos ^{2} \theta}{g^{2} a^{2} e^{x} \Omega}}\right) \partial_{\mu} \rho \partial^{\mu} \rho\right. \\
& -\mathcal{V}(\rho)),
\end{aligned}
$$

where

$$
\mathcal{V}(\rho)=4 \pi \mu_{5}\left(F^{4} a^{2} e^{3 f} \Omega E\left(\sqrt{1-\frac{e^{2 f} \cos ^{2} \theta}{g^{2} a^{2} e^{x} \Omega}}\right)+\mathcal{V}_{1}(\rho)+\mathcal{V}_{2}(\rho) \cos ^{2} \theta\right) .
$$

Canonical normalization of the inflaton field is achieved with $\rho \rightarrow \Phi$

$$
4 \pi \mu_{5} a^{2} F^{2} e^{3 f} \Omega E\left(\sqrt{1-\frac{e^{2 f} \cos ^{2} \theta}{g^{2} a^{2} e^{x} \Omega}}\right) \quad \partial_{\mu} \rho \partial^{\mu} \rho \equiv \partial_{\mu} \Phi \partial^{\mu} \Phi .
$$

In the following section we study asymptotics of the (4.4)-(4.9) that would allow the computation of the slow roll parameter $\eta$ for the model (4.22), (4.23).

\subsection{Asymptotics and a phase transition}

In this section we discuss different classes of solutions of (4.4)-(4.9). There are two topologically distinct classes of solutions of above equations:

$$
\begin{aligned}
& (a): \quad F \rightarrow F_{0}, \quad a^{2} e^{-2 f} \rightarrow z_{0}, \quad e^{-2 x} \rightarrow 0, \quad \text { as } \quad \rho \rightarrow 0 \text {, } \\
& (b): \quad F \rightarrow 0, \quad a^{2} e^{-2 f} \rightarrow z_{0}, \quad e^{-2 x} \rightarrow k_{0}, \quad \text { as } \quad \rho \rightarrow 0,
\end{aligned}
$$


where all constants $\left\{F_{0}, z_{0}, k_{0}\right\}$ are positive. What is the physical meaning of different infrared boundary conditions? The GKMW model represents a supergravity dual to $d=6 S U(N) \mathcal{N}=2$ supersymmetric Yang-Mills theory compactified (with an appropriate twist) on $S^{2}$. The 'twist' preserves half of the original supersymmetries, so that in the infrared we have $\mathcal{N}=2 S U(N)$ SYM in four dimensions. The scale of the compactification (up to a numerical factor) coincides with the strong coupling scale $\Lambda$ of the four dimensional gauge theory. As common in gauge/string duality constructions with reduced supersymmetry, decoupling the compactification scale and the scale of the strong coupling dynamics requires to go beyond the regime of validity of the supergravity approximation. Once we formulate such a gauge theory on $d S_{4}$ (or Euclidean $S^{4}$ ), the background space-time curvature (the Hubble parameter $H$ ) introduces a new infrared cutoff. One would expect now two different dynamical regimes in the gauge theory

$$
\begin{aligned}
\text { (a) : } & & \Lambda \gg H, \\
\text { (b) : } & & \Lambda \ll H .
\end{aligned}
$$

The gauge theory regimes in (4.25) are in direct correspondence with the supergravity IR boundary conditions ${ }^{10}$ (4.24). From (4.11), notice that on the supergravity side in the case $(a)$ the Euclidean gauge theory $S^{4}$ is non-contractible, while an $S^{1} \subset S^{3}$ parameterized by $\phi_{1}$ shrinks to zero size. In the case (b), the (Euclidean) gauge theory $S^{4}$ shrinks to zero size, while the squashed and twisted $S^{3}$ transverse to the five-branes remains non-contractible. On the supergravity side we explicitly demonstrate that as the compactification scale decreases with $H$ kept constant, the system undergoes a phase transitions. The physics of this transition is not clear to us. We hope to return to this problem in the future.

Let's introduce a new radial coordinate as

$$
r \equiv g \rho,
$$

${ }^{10}$ This correspondence is established by noticing that in case $(a)$ of (4.25), the limit $H \rightarrow 0$ must be smooth. The same phenomena occurs in the related model, de-Sitter deformation of the MN background [23]. 
and

$$
\begin{aligned}
& z(r) \equiv g a(r) e^{-f(r)}, \\
& f_{1}(r) \equiv e^{-2 x(r)}, \\
& F(r) \equiv \frac{H}{g} G(r),
\end{aligned}
$$

then (4.4)-(4.9) are equivalent to

$$
\begin{gathered}
0=\left[\left(G^{4}\right)^{\prime} e^{5 f} z^{2}\right]^{\prime}-12 G^{2} e^{5 f} z^{2}, \\
0=\left[\left(e^{5 f}\right)^{\prime} z^{2} G^{4}\right]^{\prime}-\frac{G^{4} e^{5 f}\left(f_{1}+8 z^{4}\right)}{2 z^{2}}, \\
0=\left[\left(\ln f_{1}\right)^{\prime} e^{5 f} z^{2} G^{4}\right]^{\prime}-\frac{f_{1} G^{4} e^{5 f}}{z^{2}}, \\
0=\left[\left(z^{2}\right)^{\prime} e^{5 f} G^{4}\right]^{\prime}+\frac{G^{4} e^{5 f}\left(f_{1}-2 z^{2}\right)}{z^{2}}, \\
+16 z G z_{1}^{2}\left(10 z f^{\prime} G^{\prime}+5 G f_{1}^{2}\left(f^{\prime}\right)^{2}-z^{2} G^{2}\left(f_{1}^{\prime}\right)^{2}+8 G^{2} f_{1}^{2}\left(z^{\prime}\right)^{2}+48 z^{2} f_{1}^{2}\left(G^{\prime}\right)^{2}\right. \\
+2 \frac{f_{1}^{2}\left(f_{1} G^{2}-4 z^{2} G^{2}-8 G^{2} z^{4}-24 z^{4}\right)}{z^{2}},
\end{gathered}
$$

where prime denote derivative with respect to $r$ as defined by (4.26).

\subsubsection{Case (a)}

Corresponding to case $(a)$ in $(4.24)$, the power series solution in the infrared is

$$
\begin{aligned}
z & =z_{0}+\frac{1}{4 z_{0}} r^{2}+\mathcal{O}\left(r^{4}\right), \\
f_{1} & =k_{0} r^{2}\left(1-\frac{\left(2 z_{0}^{2} g_{0}^{2}+6 z_{0}^{2}+g_{0}^{2}\right) r^{2}}{3 z_{0}^{2} g_{0}^{2}}+\mathcal{O}\left(r^{4}\right)\right), \\
f & =\frac{1}{5} \ln \left(h_{0}\right)+\frac{1}{5} \ln (r)+\frac{\left(4 z_{0}^{2} g_{0}^{2}-6 z_{0}^{2}-g_{0}^{2}\right) r^{2}}{30 z_{0}^{2} g_{0}^{2}}+\mathcal{O}\left(r^{4}\right), \\
G & =g_{0}+\frac{3}{4 g_{0}} r^{2}+\mathcal{O}\left(r^{4}\right),
\end{aligned}
$$

where $\left\{z_{0}, k_{0}, g_{0}\right\}$ are positive integration constants characterizing the 'size' of the wrapped $S^{2}$ in the infrared, the 'size' of the $S^{1} \subset S^{3}$ parameterized by $\phi_{1}$, in addition 
$g_{0}$ characterizes the 'size' of de Sitter space; $h_{0}$ is a trivial modulus corresponding to the value of the dilaton (4.12) in the infrared. Without loss of generality we will set $h_{0}=1$, which leaves us with the 3 -dimensional parameter space of initial conditions: $\left\{z_{0}, k_{0}, g_{0}\right\}$.

Numerically we observe that given $\left\{z_{0}, g_{0}\right\}$ there is a critical value $k_{\text {critical }}=k_{\text {critical }}\left(z_{0}, g_{0}\right)$, such that for $0<k_{0}<k_{\text {critical }}$ the supergravity solution (4.11) is singularity-free, while for $k_{0}>k_{\text {critical }}$ the background geometry has a naked time-like singularity. This singularity is associated with collapsing of the $S^{2}$ wrapped by the five-branes at finite value of the radial coordinate $r$. A typical evolution of $z(r)^{2}$ for a given set of initial parameters $\left\{z_{0}, k_{0}, g_{0}\right\}$ in (4.33) is shown in Fig. 4.

Nonsingular solutions behave asymptotically, $r \gg 1$, as

$$
\begin{aligned}
G^{2} & \rightarrow 3 r-\frac{3}{4} \ln r \\
z^{2} & \rightarrow r+\left(-\frac{1}{4}-\frac{k_{\infty}}{2}\right) \ln r \\
f & \rightarrow \frac{2}{5} r-\frac{3}{10} \ln r \\
f_{1} & \rightarrow k_{\infty}-\frac{k_{\infty}^{2}}{2} \frac{1}{r}
\end{aligned}
$$

where $k_{\infty} \equiv k_{\infty}\left(k_{0}, z_{0}\right)$ depends on the infrared data. We verified (4.34) both analytically and, by extracting relevant asymptotics, numerically. Generically $k_{\infty} \neq 1$, which implies that asymptotically the $S^{3}$ transverse to $N S 5$ branes remains squashed. It is somewhat surprising that the infrared deformation of the theory has such a profound effect on its ultraviolet properties. On the other hand, we have to remember that in the ultraviolet we are dealing with Little String Theory [44,45]. Thus it is conceivable that the observable phenomena is a reflection of the UV/IR mixing in this non-local model. This 'mixing' clearly deserves further study. 


\subsubsection{Case (b)}

Corresponding to case $(b)$ in (4.24), the power series solution in the infrared is

$$
\begin{aligned}
z & =z_{0}+\frac{2 z_{0}^{2}-k_{0}}{20 z_{0}^{3}} r^{2}+\mathcal{O}\left(r^{4}\right), \\
f_{1} & =k_{0}+\frac{k_{0}^{2}}{10 z_{0}^{4}} r^{2}+\mathcal{O}\left(r^{4}\right), \\
f & =h_{0}+\frac{8 z_{0}^{4}+k_{0}}{100 z_{0}^{4}} r^{2}+\mathcal{O}\left(r^{4}\right), \\
G & =r\left(1-\frac{4 z_{0}^{2}-k_{0}+8 z_{0}^{4}}{240 z_{0}^{4}} r^{2}+\mathcal{O}\left(r^{4}\right)\right),
\end{aligned}
$$

where $\left\{z_{0}, k_{0}\right\}$ are positive integration constants characterizing the 'size' of the wrapped $S^{2}$ in the infrared, the 'size' of the $S^{1} \subset S^{3}$ parameterized by $\phi_{1} ; h_{0}$ is a trivial modulus corresponding to the value of the dilaton (4.12) in the infrared. Without loss of generality we will set $h_{0}=0$.

Given (4.35), the two classes of solutions of (4.28) - (4.32) differ depending whether $k_{0}>k_{\text {critical }}$ or $k_{0}<k_{\text {critical }}$, for a certain ${ }^{11} k_{\text {critical }}=k_{\text {critical }}\left(z_{0}\right)$. In the former case the $S^{2}$ which $N S 5$ branes wrap starts in the infrared $r=0$ at a finite radius $z_{0}$, and ultimately collapses to zero size at finite $r=r_{\text {singular }}$, where the background has a naked time-like singularity. For $k_{0}<k_{\text {critical }}$ the supergravity flow is nonsingular, moreover as $r \rightarrow \infty$ we find the same asymptotics as in (4.34). Again, generically $k_{\infty} \neq 1$.

\subsection{Slow roll}

Given asymptotics of the nonsingular solution (4.34) (recall that the dimensionless radial coordinate $r$ is given by (4.26)), the probe brane potential (4.22) in terms of a canonically normalized inflaton field $\Phi(4.23)$ becomes

$$
\mathcal{V}(\Phi)=\frac{3}{2} H^{2}\left(1-\frac{\sqrt{k_{\infty}}}{\cos ^{2} \theta+k_{\infty} \sin ^{2} \theta}\right) \Phi^{2}\left[\ln \left(\frac{\Phi^{2} g^{6}}{\mu_{5} H^{2}}\right)+\mathcal{O}(1)\right], \frac{\Phi^{2} g^{6}}{\mu_{5} H^{2}} \gg 1,
$$

leading to generically large slow-roll parameter

$$
\eta_{G K M W}=\left(1-\frac{\sqrt{k_{\infty}}}{\cos ^{2} \theta+k_{\infty} \sin ^{2} \theta}\right)\left[\ln \left(\frac{\Phi^{2} g^{6}}{\mu_{5} H^{2}}\right)+\mathcal{O}(1)\right] .
$$

\footnotetext{
${ }^{11}$ From (4.35) it is tempting to speculate that $k_{\text {critical }}\left(z_{0}\right)=2 z_{0}^{2}$. Explicit numerical integration shows that $k_{\text {critical }}\left(z_{0}\right)<2 z_{0}^{2}$.
} 
Depending on the infrared data of supergravity phases in sections 4.3.1 and 4.3.2, nonsingular solutions can have asymptotically $k_{\infty}$ either greater of less than one, see Fig. 5. From (4.36), for $k_{\infty}>1$ the probe brane potential is locally minimized for $\sin \theta=0$, which leads to a potential unbounded from below for large values of $\Phi$. Here, the probe brane would move toward the boundary, signaling the instability encountered previously in de-Sitter deformed $\mathcal{N}=2^{*}$ warped throat geometries. For $k_{\infty}<1$ the probe brane potential is locally minimized for $\cos \theta=0$. From (4.37) it appears that fine-tuning $k_{\infty} \rightarrow 1_{-}$would lead to a slow roll inflation. This is not so, in fact for $k_{\infty}=1$ (including the subleading terms in (4.34)) we find

$$
\left.\eta_{G K M W}\right|_{k_{\infty} \rightarrow 1_{-}}=1+\frac{1}{2} \cos ^{2} \theta \ln \left[\frac{8 e}{\cos ^{2} \theta} \ln \left(\frac{\Phi^{2} g^{6}}{\mu_{5} H^{2}}\right)+\mathcal{O}(1)\right]
$$

resulting in (4.1).

\section{$5 \quad$ Phenomenology}

In this section we discuss phenomenological implications of the mobile brane inflation in de-Sitter deformed warped throats of the compactification manifold. Specifically we comment on inflation in $\mathrm{KS}$ throat [6], and de-Sitter deformed $\mathcal{N}=2^{*}$ [36], MN [21], GKMW [22] throats of the compactification manifold.

\subsection{Inflation in KS throat}

The effective four dimensional low energy description [7] and the detailed probe brane computation of [13] show that the slow-roll inflation is not possible in this simplest setup. Here, the slow role parameter is $\eta=\frac{2}{3}$. An interesting proposal to circumvent this obstacle was presented in [34], where inflation is realized by a mobile D3 brane near the enhanced symmetry point of a compactification manifold with several identical $\mathrm{KS}$ throats. One noticeable signature of the model [34] is the generic prediction for the tilt parameter $n<1$ in the spectrum of density perturbations. Current observational data indicate that for a class of models with $n<1[46,47]$

$$
n \simeq 0.97
$$

We emphasize the constraint (5.1) because in the inflationary models discussed below, thought we also would have to resort to a multiple throat geometry, we find $n>1$. 
Thus the tilt in the spectrum is a characteristic feature distinguishing our models from the one in [34].

\subsection{Inflation in de-Sitter deformed $\mathcal{N}=2^{*}$ throat}

Slow roll inflation in the de-Sitter deformed $\mathcal{N}=2^{*}$ throats was proposed in [18] and studied in details in section 2 of this paper. One noticeable difference of this setup compare to inflation in KS throat [7] is the fact that the inflationary throat does not end in the IR: the redshift factor $Z$ at the 'bottom' of the inflationary throat is exactly zero. This means that we can not generate a nonzero four dimensional Hubble parameter by placing a $\overline{D 3}$ at the bottom of the inflationary throat. Indeed, recall that in the deSitter vacua construction of KKLT [6] $H^{2} \sim Z^{4}$. A simplest resolution is to assume that compactification manifold has (as least) two warped throat geometries: one being an original KKLT throat, while inflationary one is de-Sitter deformed $\mathcal{N}=2^{*}$. A cartoon picture of this inflationary scenario is shown in Fig. 6. One might worry whether an 'infinite' in the IR $\mathcal{N}=2^{*}$ throat can be consistently 'glued' into a compactification manifold along with stabilizing the parameters that fixes $\eta$ (see Fig. 3) of the local model. We do not have a definitive answer to this question. What is clear is that the 'infinite volume' of the local de-Sitter $\mathcal{N}=2^{*}$ throat comes from its UV end (as the radial coordinate in (2.4) goes to infinity), which is expected to be cutoff very much like a volume of local KS geometry [14] is cutoff in the GKP compactification [5]. Thus, having a zero redshift at the bottom of the inflationary throat of this type does not pose an immediate obstacle for the compactification ${ }^{12}$. Also, though $\overline{D 3}$ brane at the end of the inflationary throat does not affect $H$ (which is determined by KS throat of the compactification manifold), the exit from inflation might require putting a $\overline{D 3}$ brane there anyway. In what follows we assume that a cartoon of Fig. 6 can be realized and study phenomenological implications of inflation in this model.

We assume that compactification manifold size $L$ is large in string units

$$
L^{6} \gg\left(\alpha^{\prime}\right)^{3},
$$

and

$$
g_{s} \ll 1 .
$$

Above conditions validate the use of the supergravity approximation. From the low energy effective four dimensional perspective the potential energy driving acceleration

\footnotetext{
${ }^{12}$ It is extremely interesting to rigorously establish whether such throats can be compactified.
} 
is

$$
\mathcal{V}_{e f f}(\Phi)=3 m_{p l}^{2} H^{2}+\frac{3}{2} \eta\left(\rho_{0}\right) H^{2} \Phi^{2}\left[1+\mathcal{O}\left(\frac{L^{4} H^{2} T_{3}}{\Phi^{2}}\right)\right],
$$

where the $\Phi$ independent term is a cosmological constant of the KS throat, and $\eta\left(\rho_{0}\right)$ is the slow roll parameter of mobile $D 3$ brane in the inflationary throat summarized in Fig. 3. Effective potential $\mathcal{V}_{\text {eff }}$ is computed in the approximation for a mobile brane to be far from the IR end of the inflationary throat. The latter approximation is reflected in the condition [13]

$$
\frac{L^{4} H^{2} T_{3}}{\Phi^{2}} \ll 1
$$

In (5.4) $m_{p l}$ is the four dimensional Planck constant

$$
m_{p l}^{2}=m_{10}^{8} L^{6} \sim\left(\alpha^{\prime}\right)^{-4} g_{s}^{-2} L^{6}
$$

and $T_{3}$ is Einstein frame 3 -brane tension

$$
T_{3} \sim\left(\alpha^{\prime}\right)^{-2}
$$

Finally, the computations for $\eta\left(\rho_{0}\right)$ where done in the local geometry, i.e., the mobile brane should be far from the UV end of the inflationary throat. Relating the brane position inside the throat and inflaton field $\Phi$ as in $[13,18]$ this translates into

$$
L T_{3}^{1 / 2} \gg \Phi
$$

Both conditions (5.5) and (5.8) imply

$$
H L \ll 1
$$

Notice that $H L$ is a characteristic of the KS throat of the compactification manifold.

The inflationary parameters corresponding to $\mathcal{V}_{\text {eff }}$ are [48]: slow roll parameters $\{\eta, \epsilon\}$, the tilt in the spectrum of the density perturbations $n$, the scale of the adiabatic density perturbations $\delta_{H}$, the power in the gravity wave perturbations $\mathcal{P}_{\text {grav }}$

$$
\begin{gathered}
\eta=m_{p l}^{2} \frac{\mathcal{V}_{e f f}^{\prime \prime}}{\mathcal{V}_{e f f}}=\eta\left(\rho_{0}\right), \\
\epsilon=\frac{1}{2} m_{p l}^{2}\left(\frac{\mathcal{V}_{e f f}^{\prime}}{\mathcal{V}_{e f f}}\right)^{2}=\frac{1}{2} \eta\left(\rho_{0}\right)^{2}\left(\frac{\Phi}{m_{p l}}\right)^{2}, \\
n=1-6 \epsilon+2 \eta=1-3 \eta\left(\rho_{0}\right)^{2}\left(\frac{\Phi_{i}}{m_{p l}}\right)^{2}+2 \eta\left(\rho_{0}\right),
\end{gathered}
$$




$$
\begin{gathered}
\delta_{H}=\frac{1}{\sqrt{75} \pi} \frac{1}{m_{p l}^{3}} \frac{\mathcal{V}_{e f f}^{3 / 2}}{\mathcal{V}_{e f f}^{\prime}}=\frac{1}{5 \pi \eta\left(\rho_{0}\right)} \frac{H}{\Phi_{i}}, \\
\mathcal{P}_{\text {grav }}=\frac{1}{2 \pi^{2}} \frac{H^{2}}{m_{p l}^{2}},
\end{gathered}
$$

where $\Phi_{i}$ is the value of the inflaton field $N_{e} \approx 60$ e-foldings before the end of inflation. - The first observation is that $\Phi_{i}$ must satisfy (5.8). Thus, given (5.6), (5.7) and $\eta\left(\rho_{0}\right)<1$, we have an upper bound on the slow-roll parameter $\epsilon$

$$
\epsilon \ll \eta\left(\rho_{0}\right)^{2}\left(\frac{L T_{3}^{1 / 2}}{m_{p l}}\right)^{2} \sim\left(\frac{\alpha^{\prime} g_{s}}{L^{2}}\right)^{2} \ll 1,
$$

where we used the validity of the supergravity approximation (5.2), (5.3). This immediately implies that in our model the tilt in the spectrum of perturbations is

$$
n \approx 1+2 \eta\left(\rho_{0}\right)>1
$$

Current observational data for models with $n>1$ constrain (in a $95 \%$ confindence region) $[46,47]$

$$
1<n<1.28
$$

leading to $0<\eta<0.14$. From Fig. 3 there is a large region of the allowed ${ }^{13} \rho_{0^{-}}$ parameter space (more than 60\%) consistent with this constraint.

- The slow-roll condition (assuming we adjust $\eta<0.14$ ) is valid as long as $\mathcal{V}_{\text {eff }}(5.4)$ is justified. With potential (5.4), inflation starting at $\Phi_{\text {start }}$ and ending at $\Phi_{\text {end }}$ will produce $N_{e}$ e-foldings

$$
N_{e}=\frac{1}{\eta\left(\rho_{0}\right)} \ln \left(\frac{\Phi_{\text {start }}}{\Phi_{\text {end }}}\right) .
$$

Taken for $\Phi_{\text {start }}$ and $\Phi_{\text {end }}$ the UV (5.8) and IR (5.5) cutoffs of the local inflationary throat respectively, we find that the maximal number of e-folding in this model is

$$
N_{e}^{\max } \sim-\frac{1}{\eta\left(\rho_{0}\right)} \ln (H L) .
$$

- To illustrate that phenomenologically viable scenarios are possible let's assume that $\eta=0.02$ (which is clearly possible from Fig. 3). We take

$$
\begin{aligned}
m_{p l} & =2.4 \times 10^{18} \mathrm{GeV}, \\
\alpha^{\prime-1 / 2} \equiv 1 / l_{s} & =3.5 \times 10^{15} \mathrm{GeV} .
\end{aligned}
$$

\footnotetext{
${ }^{13}$ By the construction of the model.
} 
We further assume $L \sim 5 l_{s}$. Given [46]

$$
\delta_{H}=1.9 \times 10^{-5}
$$

we find

$$
\frac{H}{\Phi_{i}} \approx 6.0 \times 10^{-6}
$$

Let's assume that inflation starts $\frac{1}{10}$ from the UV cutoff of the throat, $\Phi_{i}=\frac{1}{10} L T_{3}^{1 / 2}$. Then (5.22) leads to a low scale of inflation

$$
H \approx 6.7 \times 10^{8} \mathrm{GeV} .
$$

From (5.19) the maximum number of e-foldings in this model

$$
N_{e}^{\max } \sim 693
$$

The power in gravity wave perturbations in this model is

$$
\mathcal{P}_{\text {grav }} \approx 4.0 \times 10^{-21}
$$

which is much below the level of detection in future experiments.

\subsection{Inflation from wrapped braneworlds}

In sections 3 and 4 of this paper we considered exotic inflationary models, where a mobile $D 5$ brane was wrapping a two-cycle in the inflationary throat. These local inflationary throats are de-Sitter deformed MN geometry [23], and the newly constructed de-Sitter deformation of the GKMW background [22], respectively. In the former case we found $\eta_{M N}=\frac{3}{2}$, while in the latter $\eta_{G K M W} \geq 1$ (generically $\eta_{G K M W} \gg 1$ ). Thus in the simplest inflationary scenario advocated here, these models are excluded.

\section{Conclusion}

In this paper we discussed probe brane dynamics as a tool to study inflation in four dimensional de-Sitter vacua of string theory warped flux compactifications. This is a fruitful approach to study brane inflation in the framework proposed in [7], where the inflaton field is identified with the mobile brane position deep inside the inflationary throat. In an attempt to find slow roll single field inflationary models we 
investigated probe brane dynamics in various local de-Sitter deformed warped throat geometries. Specifically, we studied $D 3$ probe dynamics in de-Sitter deformed $\mathcal{N}=2^{*}$ throat $[39,18]$, as well as exotic inflationary models with a $D 5$ inflationary brane wrapping a two-cycle of the de-Sitter deformed MN geometry [23], or de-Sitter deformed GKMW geometry constructed in this paper. While the probe brane dynamics in local geometries can not address the question of the physics responsible for the generation of the four-dimensional Hubble scale, it has an advantage of being a rigorous analytical tool to probe the dynamics of the effective four-dimensional inflation. We found that "wrapped braneworld inflationary" models based on D5 branes wrapping a two-cycle of the resolved conifold can not lead to slow roll inflation. On the other hand, inflation from mobile $D 3$ branes in de-Sitter deformed $\mathcal{N}=2^{*}$ throats can be slow roll. Thus, it is interesting to further study the latter scenario.

In view if this, the most outstanding question is understanding the compactification of the de-Sitter deformed $\mathcal{N}=2^{*}$ throats. A possible phenomenological set-up is proposed in Fig. 6. To recapitulate, consider a Calabi-Yau threefold with fluxes generating a KS throat. These fluxes, and the compactification manifold can be chosen in such a way $[4,5]$ that the only remaining modulus would be the overall Kähler modulus of the compactification manifold. The latter can be further fixed by non-perturbative string instanton effects [6]. Further introducing a de-Sitter brane at the end of the $\mathrm{KS}$ throat can lead to a four-dimensional de-Sitter vacuum [6]. A stack of a large number of $D 3$ branes away from the KS throat of the compactification manifold would produce additional throat with zero redshift factor at the bottom ${ }^{14}$. At this stage this will be a standard $A d S_{5}$ throat with four-dimensional de-Sitter slicing and the Hubble scale as produced by the $\overline{D 3}$ brane in the KS throat. As such, slow roll inflation in this "de-Sitter deformed ${ }^{15} " \mathcal{N}=4$ throat is yet impossible. Local $\mathcal{N}=4$ throat $\left(A d S_{5} \times S^{5}\right.$ background) can be deformed into $\mathcal{N}=2^{*}$ throat (supergravity flow of Pilch and Warner [36]) by turning on 3-form fluxes and appropriately deforming the original background geometry. Likewise, local $\mathcal{N}=4$ throat with four-dimensional deSitter slicing can be deformed into de-Sitter $\mathcal{N}=2^{*}$ throat [39]. It is natural to expect that one can turn on an analogous deformation parameter (denoted $\rho_{0}$ in Fig. 3) for the stack of $D 3$ branes located on a compact manifold away from the KS throat with a

\footnotetext{
${ }^{14}$ We are assuming that sufficient number of the orientifold planes and/or 3-form fluxes is introduced to satisfy RR 5-form Bianchi identity.

15 "Deformation" here is a misnomer, as all what is required is a difference slicing of the same manifold.
} 
$\overline{D 3}$ brane sitting at its bottom. This procedure would 'compactify' the local de-Sitter $\mathcal{N}=2^{*}$ throat, inside which we argued slow roll inflation is possible. In a sense, this is parallel to the construction of GKP [5] where a local (non-compact) KS-throat was embedded inside a Calabi-Yau manifold. As a result of compactification, $\rho_{0}$, which was a parameter of the local geometry will be promoted to a dynamical field. An important question is whether dynamics is such that $\rho_{0}$ can be stabilized in the region where the slow roll is allowed, see Fig. 3.

Phenomenologically, inflation in the de-Sitter deformed $\mathcal{N}=2^{*}$ throat will be characterized by having a relatively low Hubble scale $\left(H \sim 10^{8}-10^{10} \mathrm{GeV}\right)$, negligible (unobservable) power in the gravity wave perturbations, and larger than one tilt in the spectrum of density perturbations. It will be interesting to explore models for the exit from inflation in this scenario (we mentioned that this might require placing $\overline{D 3}$ brane in the inflationary throat as well). Also, assuming that the Standard Model fields live in the KS-throat, they will not couple directly to the inflaton. Thus finding efficient mechanisms for reheating might be challenging as well.

\section{Acknowledgments}

We would like to thank Rob Myers and Mohsen Alishahiha for valuable discussions. Research at the University of Western Ontario and the Perimeter Institute is supported in part by funds from NSERC of Canada. Research at IPM is supported in part by Iranian TWAS chapter based at ISMO.

\section{References}

[1] A. Guth, "The inflationary universe: a possible solution to the horizon and flatness problems," Phys. Rev. D 23, 347 (1981)

[2] A. Linde, "A new inflationary universe scenario: a possible solutions of the horizon, flatness, homogeneity, isotropy and primodial monopole problems," Phys. Lett. B 108, 389 (1982);

[3] A. Albrecht and P. Steinhard, "Cosmology fro grand unified theories with radiatively induced symmetry breaking," Phys. Rev. Lett. 48, 1220 (1982). 
[4] S. Gukov, C. Vafa and E. Witten, "CFT's from Calabi-Yau four-folds," Nucl. Phys. B 584, 69 (2000) [Erratum-ibid. B 608, 477 (2001)] [arXiv:hep-th/9906070].

[5] S. B. Giddings, S. Kachru and J. Polchinski, "Hierarchies from fluxes in string compactifications," Phys. Rev. D 66, 106006 (2002) [arXiv:hep-th/0105097].

[6] S. Kachru, R. Kallosh, A. Linde and S. P. Trivedi, "De Sitter vacua in string theory," Phys. Rev. D 68, 046005 (2003) [arXiv:hep-th/0301240].

[7] S. Kachru, R. Kallosh, A. Linde, J. Maldacena, L. McAllister and S. P. Trivedi, "Towards inflation in string theory," JCAP 0310, 013 (2003) [arXiv:hepth/0308055].

[8] G. R. Dvali and S. H. H. Tye, "Brane inflation," Phys. Lett. B 450, 72 (1999) [arXiv:hep-ph/9812483].

[9] S. H. S. Alexander, "Inflation from D - anti-D brane annihilation," Phys. Rev. D 65, 023507 (2002) [arXiv:hep-th/0105032].

[10] G. R. Dvali, Q. Shafi and S. Solganik, "D-brane inflation," arXiv:hep-th/0105203.

[11] C. P. Burgess, M. Majumdar, D. Nolte, F. Quevedo, G. Rajesh and R. J. Zhang, "The inflationary brane-antibrane universe," JHEP 0107, 047 (2001) [arXiv:hepth/0105204].

[12] L. Randall and R. Sundrum, "An alternative to compactification," Phys. Rev. Lett. 83, 4690 (1999) [arXiv:hep-th/9906064].

[13] A. Buchel and R. Roiban, "Inflation in warped geometries," arXiv:hepth/0311154.

[14] I. R. Klebanov and M. J. Strassler, "Supergravity and a confining gauge theory: Duality cascades and chiSB-resolution of naked singularities," JHEP 0008, 052 (2000) [arXiv:hep-th/0007191].

[15] I. R. Klebanov and A. A. Tseytlin, "Gravity duals of supersymmetric SU(N) x SU(N+M) gauge theories," Nucl. Phys. B 578, 123 (2000) [arXiv:hep-th/0002159].

[16] A. Buchel and A. A. Tseytlin, "Curved space resolution of singularity of fractional D3-branes on conifold," Phys. Rev. D 65, 085019 (2002) [arXiv:hep-th/0111017]. 
[17] A. Buchel, "Gauge / gravity correspondence in accelerating universe," Phys. Rev. D 65, 125015 (2002) [arXiv:hep-th/0203041].

[18] A. Buchel, "Gauge theories on hyperbolic spaces and dual wormhole instabilities," arXiv:hep-th/0402174.

[19] A. Buchel, "On effective action of string theory flux compactifications," arXiv:hepth/0312076.

[20] A. Kehagias and E. Kiritsis, "Mirage cosmology," JHEP 9911, 022 (1999) [arXiv:hep-th/9910174].

[21] J. M. Maldacena and C. Nunez, "Towards the large N limit of pure $\mathrm{N}=1$ super Yang Mills," Phys. Rev. Lett. 86, 588 (2001) [arXiv:hep-th/0008001].

[22] J. P. Gauntlett, N. Kim, D. Martelli and D. Waldram, "Wrapped fivebranes and N = 2 super Yang-Mills theory," Phys. Rev. D 64, 106008 (2001) [arXiv:hepth/0106117]; F. Bigazzi, A. L. Cotrone and A. Zaffaroni, " $\mathrm{N}=2$ gauge theories from wrapped five-branes," Phys. Lett. B 519, 269 (2001) [arXiv:hep-th/0106160].

[23] A. Buchel, P. Langfelder and J. Walcher, "On time-dependent backgrounds in supergravity and string theory," Phys. Rev. D 67, 024011 (2003) [arXiv:hepth/0207214].

[24] H. Verlinde, "Holography and compactification," Nucl. Phys. B 580, 264 (2000) [arXiv:hep-th/9906182].

[25] C. S. Chan, P. L. Paul and H. Verlinde, "A note on warped string compactification," Nucl. Phys. B 581, 156 (2000) [arXiv:hep-th/0003236].

[26] E. Silverstein and D. Tong, "Scalar speed limits and cosmology: Acceleration from D-cceleration," arXiv:hep-th/0310221.

[27] M. Alishahiha, E. Silverstein and D. Tong, "DBI in the sky," arXiv:hepth/0404084.

[28] J. P. Hsu, R. Kallosh and S. Prokushkin, "On brane inflation with volume stabilization," JCAP 0312, 009 (2003) [arXiv:hep-th/0311077]. 
[29] H. Firouzjahi and S. H. H. Tye, "Closer towards inflation in string theory," Phys. Lett. B 584, 147 (2004) [arXiv:hep-th/0312020].

[30] L. Pilo, A. Riotto and A. Zaffaroni, "Old inflation in string theory," arXiv:hepth/0401004.

[31] M. R. Garousi, M. Sami and S. Tsujikawa, "Cosmology from rolling massive scalar field on the anti-D3 Brane of de Sitter vacua," arXiv:hep-th/0402075.

[32] C. P. Burgess, J. M. Cline, H. Stoica and F. Quevedo, "Inflation in realistic Dbrane models," arXiv:hep-th/0403119.

[33] O. DeWolfe, S. Kachru and H. Verlinde, "The giant inflaton," arXiv:hepth/0403123.

[34] N. Iizuka and S. P. Trivedi, "An inflationary model in string theory," arXiv:hepth/0403203.

[35] M. Berg, M. Haack and B. Kors, "Loop corrections to volume moduli and inflation in string theory," arXiv:hep-th/0404087.

[36] K. Pilch and N. P. Warner, "N = 2 supersymmetric RG flows and the IIB dilaton," Nucl. Phys. B 594, 209 (2001) [arXiv:hep-th/0004063].

[37] A. Buchel, A. W. Peet and J. Polchinski, "Gauge dual and noncommutative extension of an N = 2 supergravity solution," Phys. Rev. D 63, 044009 (2001) [arXiv:hep-th/0008076].

[38] N. Evans, C. V. Johnson and M. Petrini, "The enhancon and N = 2 gauge theory/gravity RG flows," JHEP 0010, 022 (2000) [arXiv:hep-th/0008081].

[39] A. Buchel, "Compactifications of the $\mathrm{N}=2^{*}$ flow," Phys. Lett. B 570, 89 (2003) [arXiv:hep-th/0302107].

[40] A. Buchel and J. T. Liu, "Thermodynamics of the $\mathrm{N}=2^{*}$ flow," JHEP 0311, 031 (2003) [arXiv:hep-th/0305064].

[41] N. Seiberg and E. Witten, "The D1/D5 system and singular CFT," JHEP 9904, 017 (1999) [arXiv:hep-th/9903224]. 
[42] J. Polchinski, "String Theory. Vol. 2: Superstring Theory And Beyond,"

[43] M. Cvetic, H. Lu and C. N. Pope, "Consistent Kaluza-Klein sphere reductions," Phys. Rev. D 62, 064028 (2000) [arXiv:hep-th/0003286].

[44] N. Seiberg, "New theories in six dimensions and matrix description of M-theory on $\mathrm{T}^{* *} 5$ and $\mathrm{T}^{* *} 5 / \mathrm{Z}(2)$," Phys. Lett. B 408, 98 (1997) [arXiv:hep-th/9705221].

[45] O. Aharony, "A brief review of 'little string theories'," Class. Quant. Grav. 17, 929 (2000) [arXiv:hep-th/9911147].

[46] C. L. Bennett et al., "First Year Wilkinson Microwave Anisotropy Probe (WMAP) Observations: Preliminary Maps and Basic Results," Astrophys. J. Suppl. 148, 1 (2003) [arXiv:astro-ph/0302207].

[47] H. V. Peiris et al., "First year Wilkinson Microwave Anisotropy Probe (WMAP) observations: Implications for inflation," Astrophys. J. Suppl. 148, 213 (2003) [arXiv:astro-ph/0302225].

[48] A. R. Liddle and D. H. Lyth, "Cosmological Inflation And Large-Scale Structure," Cambridge, UK: Univ. Pr. (2000) 400 p. 


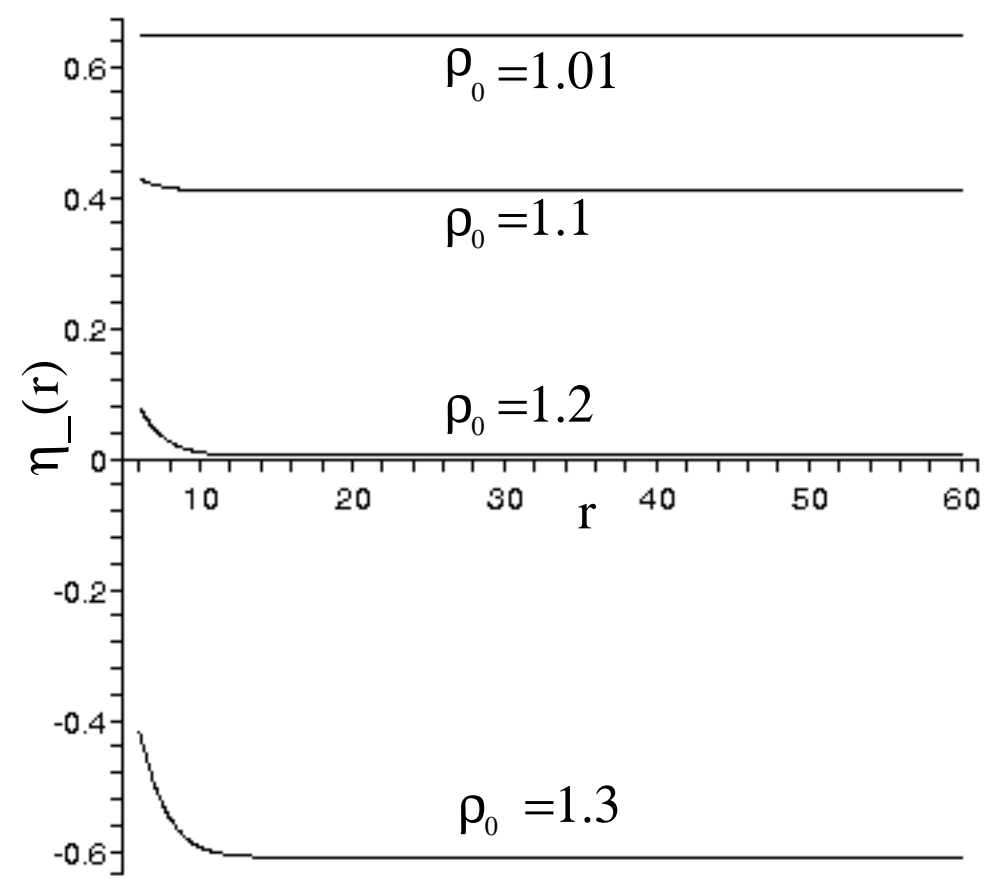

Figure 1: Numerical analysis of the slow-roll condition in de-Sitter deformed $\mathcal{N}=$ $2^{*}$ local warped geometries. The $\eta\left(\rho_{0}\right)$ parameter is the large- $r$ asymptotic of the corresponding plot $\eta\left(r, \rho_{0}\right)$. In the regime $\rho_{0}>1, \rho_{11}\left(\rho_{0}\right)<0$. Thus corresponding slow roll parameter is $\eta \equiv \eta_{-}$, defined by (2.14). Notice that with $\rho_{0}=1.3, \eta_{-}<0$, and thus from (2.13), the background is unstable. 


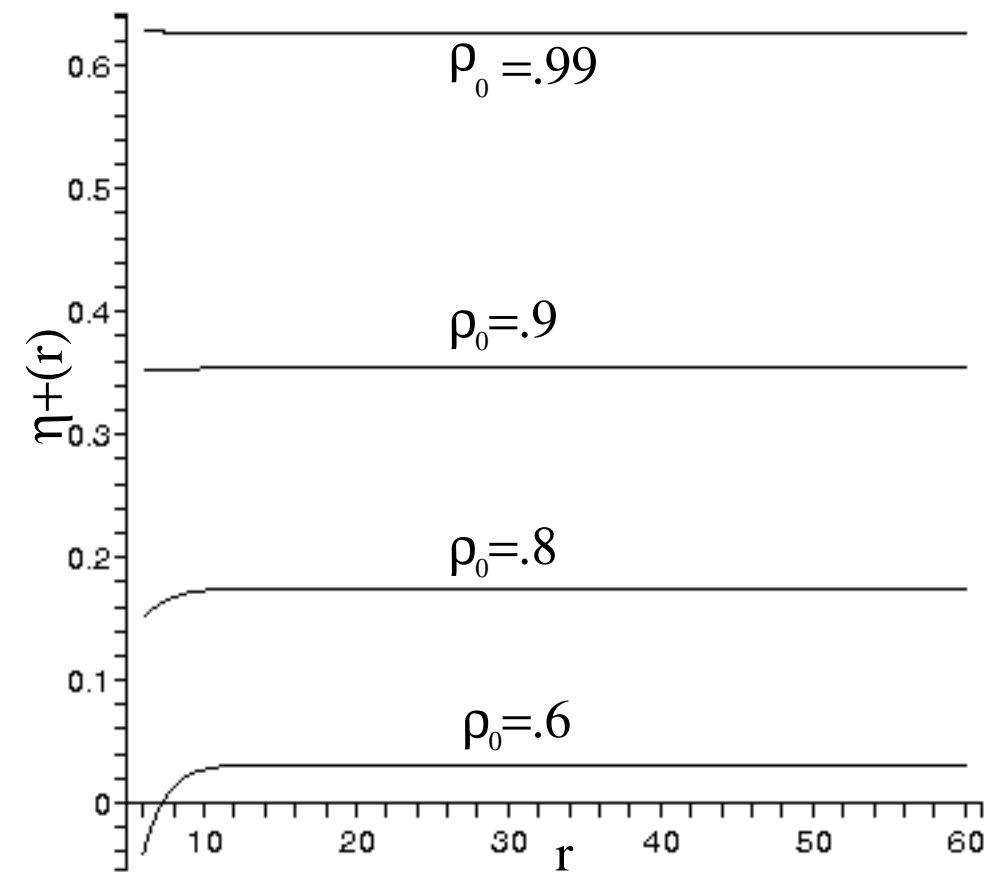

Figure 2: Numerical analysis of the slow-roll condition in de-Sitter deformed $\mathcal{N}=2^{*}$ local warped geometries. The $\eta\left(\rho_{0}\right)$ parameter is the large- $r$ asymptotic of the corresponding plot $\eta\left(r, \rho_{0}\right)$. In the regime $0 \leq \rho_{0} \leq 1, \rho_{11}\left(\rho_{0}\right) \geq 0$. Thus the corresponding slow roll parameter is $\eta \equiv \eta_{+}$, defined by (2.16). 


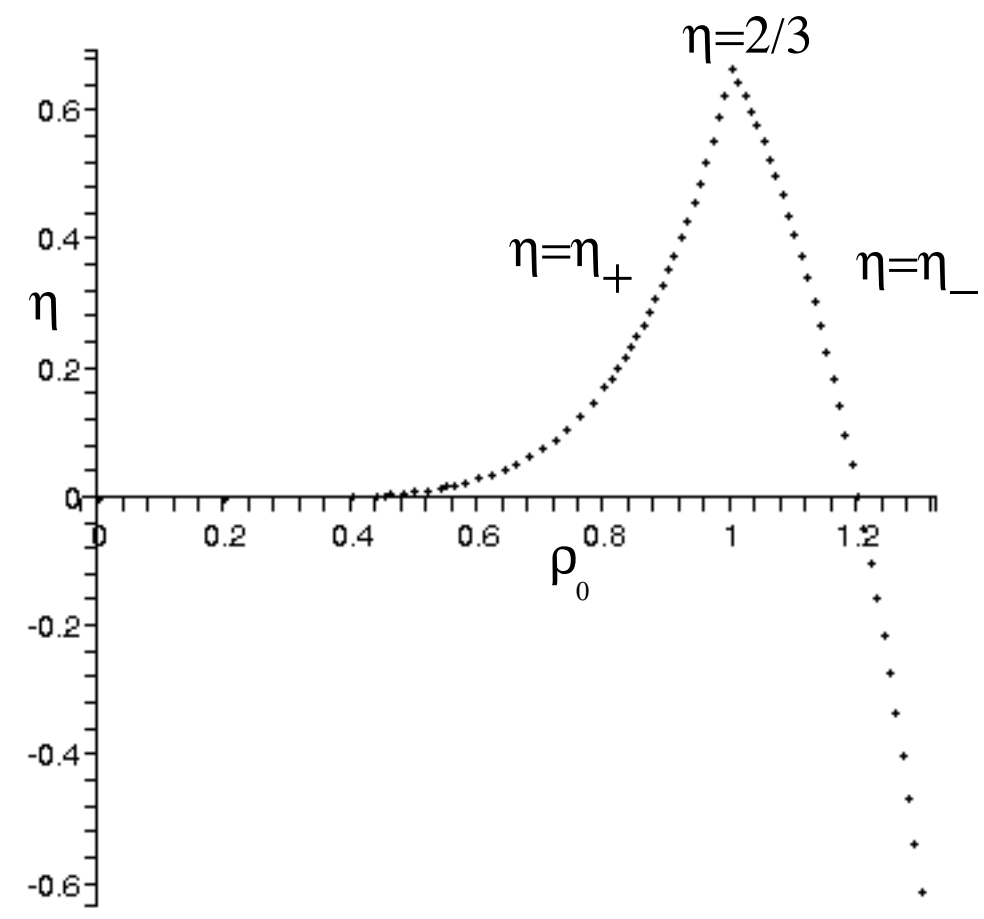

Figure 3: The slow roll parameter $\eta$ as a function of $\rho_{0}$ for $D 3$ brane inflation in deSitter deformed $\mathcal{N}=2^{*}$ throats. For $\rho_{0}>1, \eta \equiv \eta_{-}$, and $0 \leq \rho_{0} \leq 1, \eta \equiv \eta_{+}$. For $\rho_{0} \geq \rho_{\text {critical }} \approx 1.2$, we have $\eta_{-}<0$, which according to (2.13) implies the instability associated with the presence of a tachyonic mode in the spectrum of the holographically dual gauge theory. 


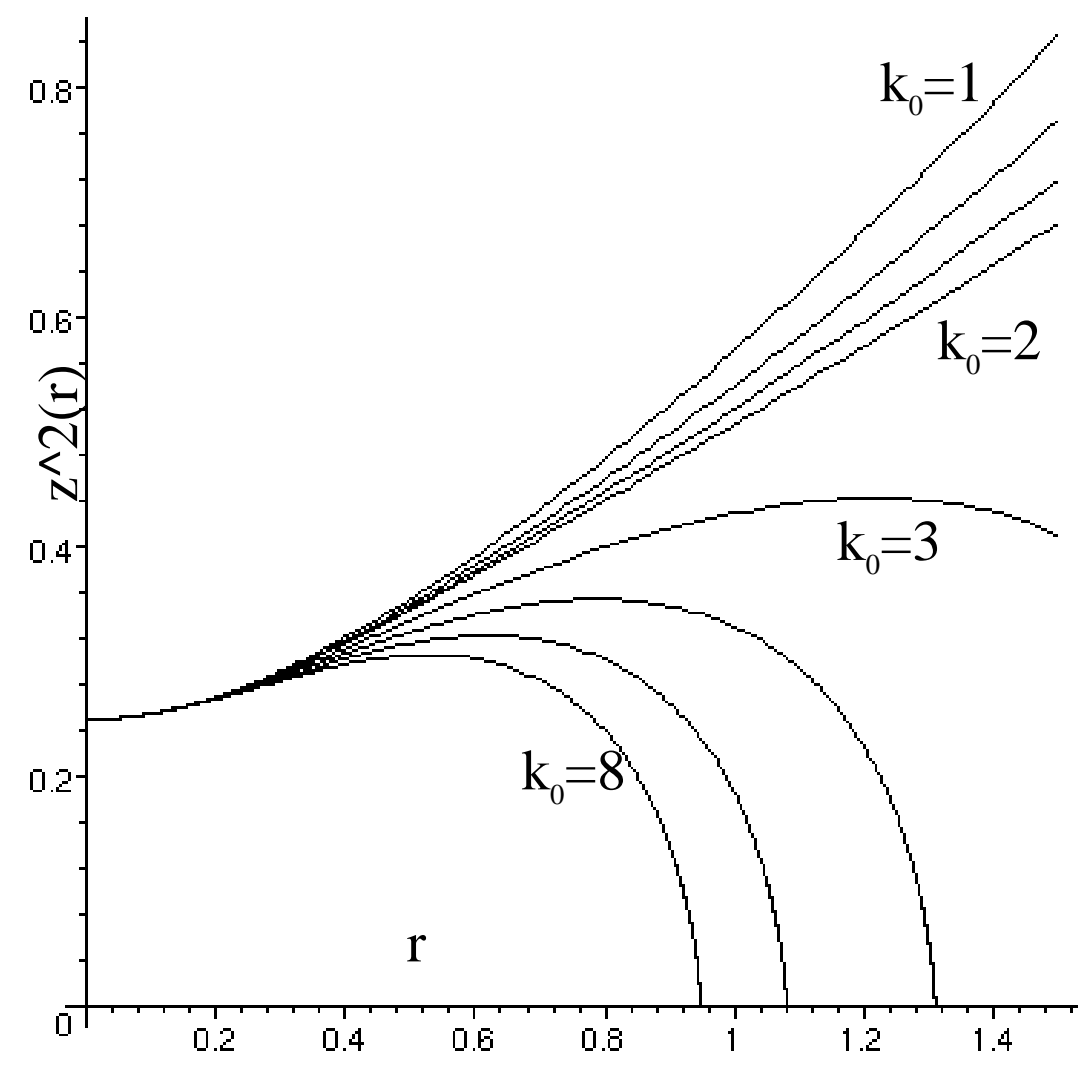

Figure 4: De-Sitter deformed GKMW solution exhibits an interesting phase transition, as one varies scales of the geometry relative to the four-dimensional Hubble parameter $H$. A typical evolution of the $S^{2}$ size, $z(r)^{2}$, wrapped by the five-branes. Here we choose infrared boundary conditions (4.33), with $g_{0}=1$, and $z(r=0) \equiv z_{0}=.5$. Notice that for $k_{0} \geq 3$ the $S^{2}$ collapses at finite $r$. One can verify that this results in a naked time-like singularity of the background geometry. For $k_{0} \leq 2$, background geometry is smooth, and the asymptotics are determined by (4.34). 


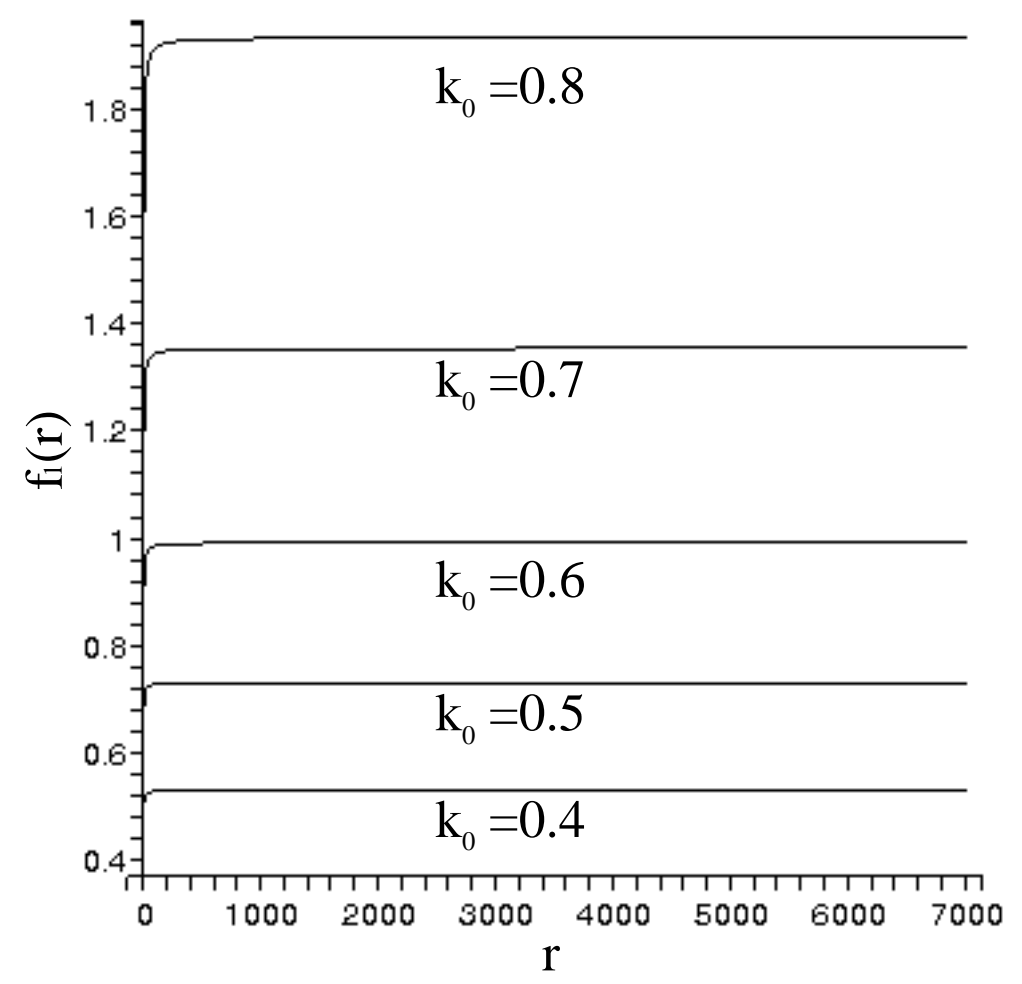

Figure 5: Large $r$ (ultraviolet) asymptotics of nonsingular solutions of both phases in the de-Sitter deformed GKMW background (4.34) are characterized by $k_{\infty} \equiv$ $\lim _{r \rightarrow \infty} f_{1}(r)$, where $f_{1}(r)$ determines the 'squashing' of the $S^{3}$ transverse to the NS5 branes. The phase (b), (4.24), asymptotic behavior of nonsingular solutions, depending on the infrared data $\left\{k_{0}, z_{0}=1\right\}$. Notice that $k_{\infty}$ can be both larger or less than one, which leads to a vastly different dynamics of the probe branes (4.36). 


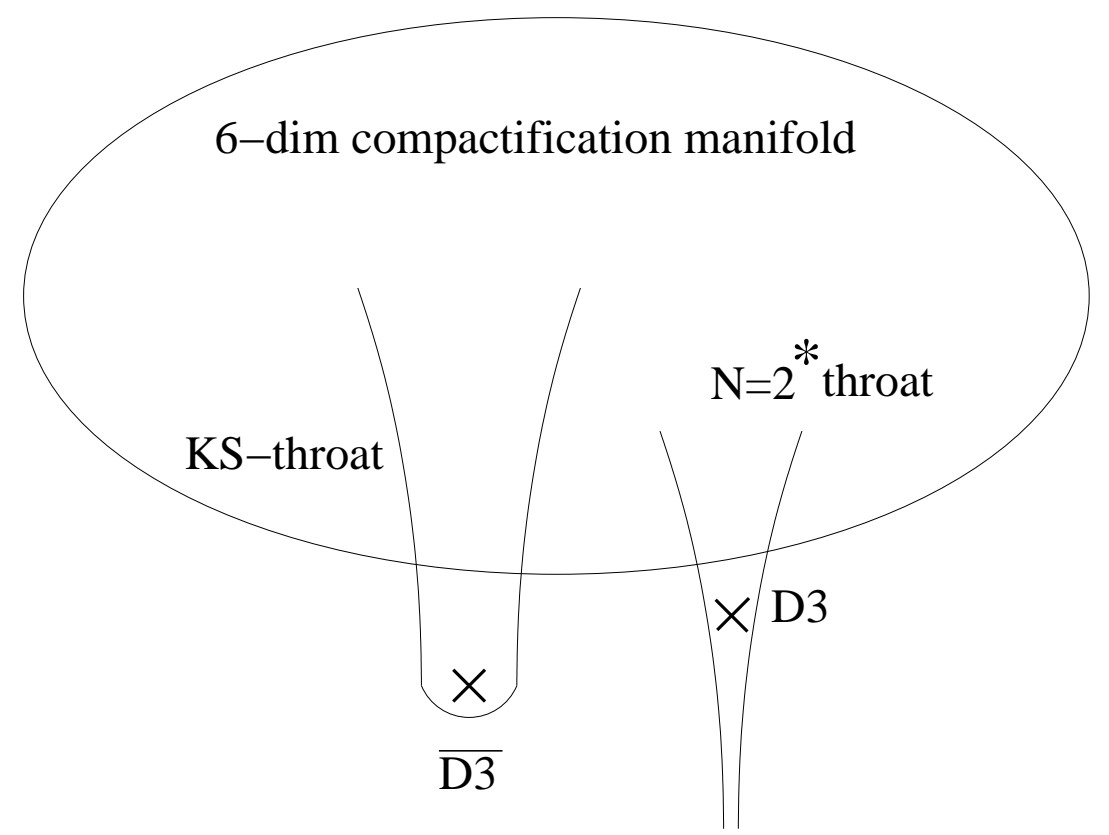

Figure 6: Inflationary scenario with a mobile $D 3$ brane in de-Sitter deformed $\mathcal{N}=2^{*}$ throat. In addition to $\mathcal{N}=2^{*}$ throat, compactification manifold is required to have a KS-throat with a $\overline{D 3}$ brane at the bottom. The Hubble scale of the four dimensional de-Sitter vacuum is set by the fluxes and the $\overline{D 3}$ in the Klebanov-Strassler throat. The slow roll inflation is realized by a mobile $D 3$ brane in $\mathcal{N}=2^{*}$ throat. 